Universidade Estadual Paulista "Júlio de Mesquita Filho" Faculdade de Medicina Campus de Botucatu

\title{
Pico e flutuação da pressão intra-ocular:
}

Comparação entre curva tensional diária e teste de sobrecarga hídrica e Comparação entre 2 testes de sobrecarga hídrica em horários diferentes.

Dissertação apresentada ao Programa de Bases Gerais da Cirurgia, Faculdade de Medicina de Botucatu- Universidade Estadual Paulista- UNESP para obtenção do título de Mestre em Oftalmologia

\section{Mariluci Tosi Addad}

Orientador: Prof. Dr. César Tadeu Spadella

Co- Orientadora: Prof. Dra. Maria Rosa Bet de Moraes Silva 
FICHA CATALOGRÁFICA ELABORADA PELA SEÇÃO TÉCNICA DE AQUISIÇÃO E TRATAMENTO DA INFORMAÇÃO

DIVISÃO TÉCNICA DE BIBLIOTECA E DOCUMENTAÇÃO - CAMPUS DE BOTUCATU - UNESP BIBLIOTECÁRIA RESPONSÁVEL: Selma Maria de Jesus

\section{Addad Mariluci Tosi.}

Pico e flutuação da pressão intra-ocular: comparação entre curva tensional diária e teste de sobrecarga hídrica e comparação entre 2 testes de sobrecarga hídrica em horários diferentes / Mariluci Tosi Addad. - Botucatu : [s.n.], 2010.

Dissertação (mestrado) - Faculdade de Medicina de Botucatu, Universidade Estadual Paulista, 2010.

Orientador: César Tadeu Spadella

Co-orientadora: Maria Rosa Bet de Moraes Silva

Assunto CAPES: 40101177

1. Glaucoma - Testes 2. Olhos - Doenças

Palavras-chave: Curva tensional diária; Glaucoma primário de ângulo aberto; Hipertensão ocular; Pico e flutuação de PIO; Teste de sobrecarga hídrica; 
Dedicatóría 
A DEUS por ter-me conduzido na solução de situações aparentemente impossiveis.

A mew pai José Carlos, que além de ter me guiado na profissão, é um exemplo de vida, está sempre ao meu lado, me conduz e itumina os caminhos por ando sigo.

A minha mãe Maria de Lourdes, irmã Mariângela, sobrinhos Matheus e Alice pelas muitas alegrias proporcionadas nestes anos de crescimento e apoio emocional nos momentos necessários.

Ao meu marido Daniel pelo amor, paciencia, incentivo e companhia em todos of momentos ao longo desta caminhada. 
Agradecimentos Especiais 
Ao Prof. Dr. César Tadeu Spadella, orientador, pelo apoio prestado, que me dew forças para continuar mew percurso, meu agradecimento será eterno.

A Profa. Dra. Dra Maria Rosa Bet de Moraes Súlva, co-Orientadora pela amizade, profissionalismo incontestável, dedicação, sabedoria e paciência. Seus ensinamentos sempre serão lembrados. 
Agradecimentos 
As colaboradoras, Sra. Cinthía Scolástico Cecílio e Núlse Ribeiro da Silva da secretaria do departamento de oftalmologia e otorrinolaringologia e Círurgia de Cabeça e Pescoço pelo profissionatismo e pela presteza que sempre me atenderam.

Aos docentes da disciplina de Oftalmololgia da FMB, por poder desenvolver a parte prática do trabalho.

Aos enfermeiros, técnicos e auxiliares de enfermagem da enfermaria de Oftalmologia e Otorrinolaringologia do HC de Botucatu, pela colaboração durante a reatização dos exames dos pacientes internados.

Aos pacientes que se dispuseram a participar do estudo, colaborando com a ciéncía.

Ao GAP (Grupo de Apoí à Pesquisa), em especial ao Hélio Rubens de Carvalho Nunes da Faculdade de Medicina de Botucatu- UNESP, pelo análise estatistica.

A bibliotecáría Rosemary Cristina da Sílva da biblioteca da UNESP, pela revisão das referências bibliográficas.

A funcionáría da biblioteca Rosângela Antônio da biblioteca do Hospital Estadual de Bauru, que me auxitiou nas buscas de inimeros trabalhos.

Aos colegas do Tosi Instituto Oftalmológico de Bauru, Dr. José Carlos Tosi, Dr. Paulo Fernando Domeneghett, pela retaguarda nestes anos de atividade acadêmicas.

As colaboradoras do Tosi Instituto Oftalmológico de Baurw: Fernanda Soares Coelho, Marilza Borges, Mary Angélica Moço, Eliane Dias e Helena Pereira da Silva, peto indispensável apoio. 
Epigrafe 
"É melhor atirar-se à luta em busca de dias methores, mesmo correndo $\sigma$ risco de perder tudo, do que permanecer estático, como os pobres de espírito, que não lutam, mas também não vencem, que não conhecem a dor da derrota, nem a glória de ressurgir dos escombros. Esses pobres de espírito, ao final de sua jornada na Terra não agradecem a Deus por terem vivido, mas desculpam-se perante Ele, por terem apenas passado pela vida."

Bof Marley 
Sumárío 
Resumo

Capítulo I

Comparação de pico e flutuação de PIO entre 2 TSH realizados em horários diferentes

Resumo

Abstract

Introdução

Objetivos

Material e Métodos

Resultados

Discussão

Conclusões

Referências Bibliográficas

Capítulo II

Comparação de pico e flutuação de PIO entre curva tensional diária e teste de sobrecarga hídrica.

Resumo

Abstract. 50

Introdução.

Objetivos

Material e Métodos.

Resultados. 60

Discussão 64

Conclusões. 68

Referências Bibliográficas 69

Anexos. 
Resumo 
TíTULO: Pico e flutuação da pressão intra-ocular - Comparação entre curva tensional diária e teste de sobrecarga hídrica e comparação entre 2 testes de sobrecarga hídrica em horários diferentes. OBJETIVOS: Comparar pico e flutuação da pressão intra-ocular (PIO) entre curva tensional diária (CTD) e teste de sobrecarga hídrica (TSH) e entre $2 \mathrm{TSH}$ em horários diferentes. MATERIAL E MÉTODOS: 29 olhos de 29 pacientes portadores de glaucoma primário de ângulo aberto ou hipertensão ocular foram submetidos à CTD e TSH, com paciente internado. A PIO foi avaliada, com tonômetro de Goldmann às $9,12,15,18,21,24$ e $6 \mathrm{~h}$, com paciente sentado. As medidas das 3 e 6 horas da manhã foram realizadas no leito com tonômetro de Perkins. Imediatamente após a medida no leito deitado, foi realizada outra medida das 6 horas com o paciente sentado. No TSH os pacientes ingeriam 1 litro de água, em até 5 minutos. A PIO foi avaliada imediatamente antes e durante uma hora após a ingestão de água, com intervalos de 15 minutos. O TSH foi realizado logo após a avaliação da $\mathrm{PIO}$ às $6 \mathrm{~h}$ da manhã e foi repetido às 14 horas. Foram avaliados em cada um dos testes: PIO basal, pico e flutuação. Foram calculados valores de média e desvio padrão para PIO basal e pico, mediana e quartís para: flutuação (também foi calculada a porcentagem). Para comparação de médias foi realizado o teste $t$ de Student e teste de Wilcoxon para comparação de medianas entre amostras dependentes. Foi considerado nível de significância $p<0,05$. RESULTADOS: A média dos valores de picos de PIO foi significativamente maior na CTD $(20,39 \pm 1,03 \mathrm{mmHg})$ do que no TSH $(17,37 \pm 0,78 \mathrm{mmHg}), \quad p<0,001$. A média dos valores de flutuação também foi maior na CTD $(10,17 \pm 0,90)$ do que no TSH $(5,0 \pm 0,45), p<$ 
0,001. O valor da flutuação em percentil na CTD foi de $90,46 \%,(70,00 ; 129,90)$ enquanto que no TSH foi de 35,42\%, (27,92;56,95), p $<0,001$.No TSH realizado às 6 horas da manhã a média dos valores de PIO basal foi de 14,31 $\pm 3,38 \mathrm{mmHg}$ e no TSH realizado às 14 horas foi de $12,72 \pm 3,5 \mathrm{mmHg}$. A comparação entre esse dois valores mostrou diferença estatisticamente significativa $(p=0,022)$. A média dos valores do pico de PIO foi semelhante nos dois $\mathrm{TSH}, 17,34 \pm 4,52 \mathrm{mmHg}$ e de $16,79 \pm 3,76 \mathrm{mmHg}$, respectivamente às 6 e às 14 horas, $(p=0,431)$. A mediana da flutuação em ambos TSH foi de 5,00 $m m H g(p=0,385)$. Em porcentagem, a mediana da flutuação foi de $33,33 \%$ no TSH das 6 horas da manhã, e de 40,00\%, no TSH das 14 horas, porém, com a diferença não significativa ( $p=0,127)$. CONCLUSÃO: Pico e flutuação de PIO na CTD foram maiores que os do TSH. Tanto o pico, como a flutuação da PIO, nos TSH da manhã e da tarde tiveram valores semelhantes, assim o TSH pode ser realizado em qualquer horário do dia.

PALAVRAS-CHAVE: glaucoma primário de ângulo aberto + teste de sobrecarga hídrica + hipertensão ocular + pico e flutuação de PIO + curva tensional diária 
Capitulo I Comparação de pico e futuaça de PIO entre curva tensional diaria e teste de sobrecarga hidrica 


\section{Comparação de pico e flutuação de PIO entre curva tensional diária e teste de sobrecarga hídrica}

\section{RESUMO}

OBJETIVOS: Comparar pico e flutuação de pressão intra-ocular (PIO) entre curva tensional diária e teste de sobrecarga hídrica, detectar os horários de pico de PIO, comparar os valores da PIO nos horários das 6 horas da manhã deitado e sentado. MATERIAL E MÉTODOS: 30 olhos direitos de 30 pacientes portadores de glaucoma primário de ângulo aberto (GPAA) ou hipertensão ocular (HO), foram submetidos à curva tensional diária de 24 horas (CTD) e teste de sobrecarga hídrica (TSH). Foram avaliados: pico (maior valor da PIO durante a CTD e TSH ) e flutuação (maior valor da PIO menos o menor valor da PIO durante o CTD e TSH). A PIO foi avaliada, com tonômetro de aplanação de Goldmann às $9,12,15,18,21,24$ e $6 \mathrm{~h}$, com paciente sentado. As medidas das 3 e 6 horas da manhã foram realizadas no leito com tonômetro de Perkins com o paciente em posição supina (deitado). Imediatamente após a medida no leito deitado, foi realizada outra medida das 6 horas com o paciente sentado. Para realização do TSH, os pacientes ingeriam 1 litro de água, em até 5 minutos. A PIO foi avaliada imediatamente antes e durante uma hora após a ingestão de água, com intervalos de 15 minutos entre as medidas. $\mathrm{O}$ TSH foi realizado logo após a avaliação da PIO às $6 \mathrm{~h}$ da manhã. Foram calculados valores de média (M) e desvio padrão (s) para pico e flutuação e mediana (Md) e quartís (Q2)

para flutuação em porcentagem (\%). Para comparação de médias foi realizado 
o teste t de Student para amostras dependentes. Foi utilizado teste de Wilcoxon para comparação de medianas entre amostras dependentes. Para todas as análises foi considerado nível de significância $p<0,05$. RESULTADOS: A média dos valores de pico de PIO foi significativamente maior na CTD $(20,39$ $\pm 1,03 \mathrm{mmHg})$ do que no TSH $(17,37 \pm 0,78 \mathrm{mmHg}), \quad p<0,001$.A média dos valores de flutuação também foi maior na CTD $(10,17 \pm 0,90)$ do que no TSH $(5,0 \pm 0,45), p<0,001$. O valor da flutuação em percentil na CTD foi de 90,46\%, $(70,00 ; 129,90)$ enquanto que no TSH foi de 35,42\%, $(27,92 ; 56,95), p<0,001$. A PIO das $6 \mathrm{~h}$ da manhã mostrou diferença estatisticamente significativa quando realizada com o paciente deitado $(17,47 \mathrm{mmHg})$ do que sentado $(14,30 \mathrm{mmHg})$, ( $p$ < 0,001). CONCLUSÕES: O TSH subestima pico e flutuação da PIO em pacientes portadores de GPAA e HO. A maior incidência de pico de PIO ocorreu às 3 horas da manhã (40\%). Houve influencia postural na avaliação das 6 horas da manhã. O melhor horário para detecção de pico de PIO é em ordem decrescente: 3 horas da manhã, 6 horas da manhã deitado e 6 horas da manhã, sentado.

PALAVRAS-CHAVE: pico e flutuação de PIO + glaucoma primário de ângulo aberto + hipertensão ocular+ teste de sobrecarga hídrica + curva tensional diária 


\section{Comparison IOP peak and fluctuation between the water drinking test and diurnal tension curve}

\section{ABSTRACT}

PURPOUSE: Comparing peak and fluctuation of intraocular pressure (IOP) between diurnal tension curve and water drinking test, detect the peak of IOP, compare the IOP at 6AM in supine and sitting position. METHODS: 30 right eyes of 30 glaucoma patients with open angle glaucoma (OAG) or ocular hypertension $(\mathrm{HO})$ were submitted to water drinking test (WDT) and diurnal tension curve (DTC). Were evaluated: peak (higher value of IOP observed during WDT and DTC), fluctuation (difference between highest and lowest value of IOP WDT and DTC). IOP was measured with applanation Goldmann tonometry at 9AM, 12AM, 3PM, 6PM, 9PM, 12PM and $6 \mathrm{AM}$, with the patient sitting. Measurements of $3 A M$ and $6 A M$ were made in bed with Perkins tonometer with the patient in the supine position. Immediately after the measure in supine position, another measure was done at $6 \mathrm{AM}$, with the patient seated. The WDT involved ingestion of 1 liter of tap water in 5 minutes. IOP was measured immediately before and one hour after ingestion of water at intervals of 15 minutes between measurements. The WDT was performed immediately after the measure IOP at 6 AM. For comparison of means was performed Student's t test for dependent samples. Wilcoxon test was used to compare medians between dependent samples. The level of significance was considered $p<0.05$. RESULTS: The mean peak IOP was significantly higher 
in the DTC $(20.39 \pm 1.03 \mathrm{mmHg})$ than in TSH $(17.37 \pm 0.78 \mathrm{mmHg}), p<0.001$. The mean fluctuation was also higher in the DTC $(10.17 \pm 0.90)$ than in WDT $(5.0 \pm 0.45), p<0.001$. The value of the fluctuation in the DTC percentile was $90.46 \%(70.00,129.90)$ while the WDT was $35.42 \%(27.92,56.95), p<0.001$. The IOP of 6 AM showed a statistically significant when performed with the patient in supine position $(17,47 \mathrm{mmHg})$ and sitting $(14,30 \mathrm{mmHg})$ and was significantly higher with the patient in supine position. $(p<0,001)$. CONCLUSION: The WDT peak and underestimates of IOP fluctuation in patients with OAG and $\mathrm{HO}$. The highest incidence peak occurred at 3 o'clock in the morning $(40 \%)$. There were influences postural evaluation from 6 o'clock in the morning. The best time to detection of peak IOP is in descending order: 3 o'clock, 6 o'clock in the morning, lying and 6 o'clock in the morning, sitting

KEYWORDS: peak and fluctuation IOP +water drinking test+ diurnal tension curve+ open angle glaucoma + ocular hypertension 


\section{INTRODUÇÃO}

O glaucoma é a principal causa de cegueira irreversível no mundo. Segundo a Organização Mundial de Saúde é o responsável por 12,3\% da cegueira mundial, atrás apenas da catarata, sendo essa causa reversível ${ }^{1}$. Estima-se que para o ano de 2010 haja 60,5 milhões de portadores da doença, sendo 4,5 milhões cegos bilateralmente ${ }^{2}$. Entretanto, a cegueira é evitável, em quase todos os casos e depende de detecção precoce e tratamento adequado $^{3}$.

O glaucoma é uma neuropatia óptica crônica, progressiva, caracterizada por alterações típicas do disco óptico e da camada de fibras nervosas da retina, com repercussões características no campo visual. É acompanhado, na maioria das vezes, de pressões intra-oculares (PIO) acima de níveis considerados estatisticamente normais ${ }^{4}$.

A elevação da PIO é o mais importante fator de risco para desenvolvimento e progressão da doença ${ }^{5-8}$. A redução da PIO é o único tratamento existente para prevenção da neuropatia óptica glaucomatosa ${ }^{9-11} \cdot \mathrm{Na}$ década passada vários estudos multicêntricos apontaram a importância do controle da PIO em pacientes portadores de glaucoma ${ }^{7,12-16}$.

Alguns estudos apontam que a PIO média ${ }^{7,9,12,13,15,18}$, é responsável pela progressão do glaucoma ou conversão para glaucoma ${ }^{19}$, outros consideram a flutuação ou picos de PIO como fatores independentes ${ }^{6,20-25}$. 
A redução da PIO média, nos estudos citados, apesar de benéfica não impediu a conversão para glaucoma ou progressão da doença em todos dos pacientes. Isto sugere que a PIO média não foi suficientemente reduzida ou que outros fatores estariam envolvidos na conversão ou progressão, como picos de PIO e flutuação ${ }^{6,17,26}$. Pacientes com a PIO aparentemente controlada em horário de consultório, podem apresentar evolução do glaucoma $^{13,16,21,27,28}$.A PIO sofre variações fisiológicas ao longo do dia ${ }^{29-32}$.

Em indivíduos normais os valores da flutuação diária variam de 3 a 6 $\mathrm{mmHg}^{33}$ e essas variações costumam ser maiores em pacientes glaucomatosos. Sendo assim, o estudo da PIO ao longo do dia é fundamental para avaliação de pacientes glaucomatosos, com perda de campo visual ou progressão no nervo óptico, para controle de tratamento em pacientes em uso de medicação, em casos de dúvida de diagnóstico ${ }^{34}$ e em alguns casos, até modificação da conduta ${ }^{29}$.

A avaliação de 24 horas pode ser feita pela curva tensional diária (CTD), que usualmente é realizada medindo a $\mathrm{PIO}$, com intervalo de $3 / 3 \mathrm{~h}$, num total de 7 medidas, das 9 às 6 horas no leito. A CTD permite a avaliação dos picos pressóricos e flutuação da PIO. Entretanto tem sido pouco utilizada pelas dificuldades inerentes à sua realização, que requer a internação do paciente, causa desconforto e altera a rotina do paciente e é dificultosa para o médico. Diante das dificuldades da realização da CTD, tem-se buscado métodos alternativos que detectem picos e flutuações, tais como, a minicurva e o teste de sobrecarga hídrica (TSH). 
A minicurva normalmente é realizada nos horários habituais de consultório, porém não detecta picos, pois estes ocorrem na sua maioria na madrugada ou nas primeiras horas do $\mathrm{dia}^{27,29,35,36}$. Outra maneira de avaliar picos e flutuações é o TSH ${ }^{11,37-40}$.

Os picos e flutuações de PIO do TSH tem sido comparado com os da minicurva (medidas feitas em horários de consultório, normalmente com 4 medidas de PIO, das 8 até 17 horas) $)^{11,37,41,42}$ ou com a CTD incompleta (medidas feitas durante o dia, até 20 horas) ${ }^{38}$. Medeiros et $\mathrm{al}^{38}$ observaram forte correlação entre os valores de pico de PIO na CTD incompleta e TSH, de 20,8 e 21,1 $\mathrm{mmHg}$, respectivamente, em pacientes glaucomatosos, em tratamento.

Os estudos que compararam o pico e flutuação de PIO na CTD completa com o TSH apesar de indicarem correlação, mostram discrepâncias grandes entre os valores ${ }^{43}$ e há contradições de qual exame seria mais eficaz $^{44,45}$.

Diante das controvérsias da literatura foi realizado o presente estudo para comparar pico e flutuação da CTD com o TSH. 


\section{OBJETIVOS}

1. Comparar pico e flutuação de pressão intra-ocular (PIO) entre CTD e TSH,

2. Detectar os horários de pico de PIO na CTD,

3. Comparar os valores da PIO nos horários das 6 horas da manhã deitado e sentado.

\section{MATERIAL E MÉTODOS}

Foi feito estudo prospectivo em 30 olhos direitos de 30 pacientes portadores de glaucoma primário de ângulo aberto ou hipertensão ocular do Ambulatório de Glaucoma do Hospital das Clínicas da Faculdade de Medicina de Botucatu- UNESP. O estudo foi realizado no período de 06 de janeiro à 07 setembro de 2007. Todos os pacientes concordaram em participar do estudo, após explanação do mesmo e assinaram o termo de consentimento Livre e Esclarecido. Foi aprovado pelo Comitê de Ética da Faculdade de Medicina de Botucatu, CEP número 2397/2007.

Todos os pacientes foram submetidos a exames de acuidade visual, refração, biomicroscopia, gonioscopia, fundoscopia e perimetria computadorizada. 
Os pacientes que estavam em uso de medicação tópica e sistêmica, mantiveram o uso regular das mesmas.

Foram incluídos neste estudo os pacientes que preenchiam os seguintes critérios de inclusão:

- Disco óptico com duas ou mais das alterações glaucomatosas: assimetria $>0,2$ entre as escavações dos dois olhos, presença de defeito em cunha, perda do padrão ISNT, vasos em baioneta, hemorragia no disco óptico e sinal de Hoyt.

- Campo visual com pelo menos: 3 pontos adjacentes não periféricos com redução $\geq 5 \mathrm{~dB}$ no gráfico pattern deviation, sendo que um deles com $\geq 10 \mathrm{~dB}$, em localização compatível com defeito glaucomatoso.

- Ausência de doença ocular ou doença neurológica que justificasse alteração de campo visual.

- $\mathrm{PIO}>$ a $21 \mathrm{mmHg}$, sem alteração de campo visual ou disco óptico (hipertensos oculares).

- Ângulo aberto e de aspecto normal, à gonioscopia (classificação grau 3 e 4 de Shaffer).

Foram excluídos do estudo:

- Pacientes do sexo feminino com risco de gravidez,

- Pacientes que realizaram cirurgia ocular e tratamentos a laser há menos de 6 meses. 
- Pacientes com acuidade visual $<20 / 40$.

- Pacientes portadores de patologia de córnea, úvea ou qualquer outra patologia que pudesse alterar a PIO.

- Pacientes portadores de Síndrome de pseudoexfoliação, com ou sem glaucoma.

- Usuários de medicação sistêmica que pudesse interferir na PIO.

- Pacientes que não conseguissem completar todos os exames.

- Pacientes com patologia renais crônicas e insuficiência cardíaca.

A medida da acuidade visual foi feita com tabela de Snellen, com melhor correção óptica, estando o paciente a 6 metros da mesma.

A refração foi realizada com refrator automático (modelo Accuref $\mathrm{K}$, Shin- Nippon, Tókio), sem ciclopegia e foi seguida da refração dinâmica no refrator. Nos pacientes com menos de 40 anos, também foi realizada refração estática.

A gonioscopia foi realizada com a lente de Goldmann de 1 espelho, após anestesia tópica e com auxílio de metilcelulose $2 \%$.

A biomicroscopia do segmento anterior foi feita com lâmpada de fenda e foram avaliadas e descritas alterações nas pálpebras, córnea, câmara anterior, íris e cristalino.

A biomicroscopia do fundo do olho foi feita, após dilatação, com colírio de tropicamida $1 \%$ (Midriacyl $1 \%{ }^{\circledR}$ ), com auxílio de lente de Volk de 78 
dioptrias. Foi realizada por 2 examinadores e as alterações foram descritas e comparadas.

O campo visual foi realizado com perímetro computadorizado Humphrey Field Analyser (modelo 740, San Leandro, Califórnia, USA), usando estratégia 24-2, e correção óptica adequada. Foi analisado por 2 examinadores.

As pressões intra-oculares foram avaliadas com o tonômetro de Goldmann (Haag Streit- Berna- Suíça) acoplado à lâmpada de fenda, previamente calibrado. Antes da tonometria, instilou-se 1 gota de colírio preparado: $1 \mathrm{ml}$ de fluoresceína sódica 1\% (Fluoresceína sódica $1 \%{ }^{\circledR}$ ) diluído em 1 frasco de proximetacaína 0,5\% $\left(\right.$ Anestalcon $\left.{ }^{\circledR}\right)$.

Para avaliação de pico e flutuação de PIO os pacientes foram submetidos à curva tensional diária (CTD) e teste de sobrecarga hídrica (TSH), com paciente internado. A PIO foi avaliada, com tonômetro de aplanação de Goldmann às $9,12,15,18,21,24$ e $6 \mathrm{~h}$, com paciente sentado. As medidas das 3 e 6 horas da manhã foram realizadas no leito com tonômetro de Perkins com o paciente em posição supina. Após as 24 horas era solicitado ao paciente não se levantasse e a luz do quarto permanecesse apagada. Imediatamente após a medida no leito deitado, foi realizada outra medida das 6 horas com o paciente sentado. O intervalo entre as medidas das 6 horas deitado e sentado não foi superior a 10 minutos.

O teste de sobrecarga hídrica (TSH) foi realizado após jejum mínimo de 2 horas de sólidos e líquidos. Os pacientes ingeriam 1 litro de água, na temperatura ambiente em até 5 minutos. A PIO foi avaliada imediatamente 
antes e durante uma hora após a ingestão de água, com intervalos de 15 minutos entre as medidas. O teste foi realizado logo após a avaliação da PIO às 6h da manhã.

Todas as medidas do TSH e CTD foram feitas pelo mesmo examinador, que desconhecia o diagnóstico dos pacientes.

As PIO foram avaliadas com o tonômetro de Goldmann (Haag Streit, Berna, Suíça) acoplado à lâmpada de fenda, previamente calibrado. Antes da tonometria, instilou-se 1 gota de colírio preparado: $1 \mathrm{ml}$ de fluoresceína sódica 1\% (Fluoresceína sódica $1 \%{ }^{\circledR}$ ) diluído em 1 frasco de proximetacaína $0,5 \%$ $\left(\right.$ Anestalcon ${ }^{\circledR}$ ), da mesma forma com o tonômetro de Perkins, no leito.

Considerou-se pico: o maior valor da PIO observado durante o TSH e CTD.

Considerou-se flutuação o maior valor da PIO menos o menor valor da PIO nos TSH e CTD.

Foram calculados valores de média (M) e desvio padrão (s) para pico e flutuação mediana (Md) e quartís (Q2) para flutuação em porcentagem (\%).

Para comparação de médias foi realizado o teste $t$ de Student para amostras dependentes. Foi utilizado teste de Wilcoxon para comparação de medianas entre amostras dependentes. Foi utilizado o programa SPSS v. 15.0. para análise estatística. Para todas as análises foi considerado nível de significância $p<0,05$. 


\section{RESULTADOS}

A média de idade dos 30 pacientes incluídos no estudo foi de 60,61 $\pm 13,4$ anos, sendo $20(66,66 \%)$ brancos e $10(33,34 \%)$ pacientes pardos, negros e amarelos. Eram 16 homens e 14 mulheres. Dos olhos analisados, 15 (50\%) eram hipertensos oculares e 15 (50\%) olhos tinham glaucoma primário de ângulo aberto sendo que 12 em uso de uma medicação e 3 estavam em tratamento com 2 ou mais colírios.

A média dos valores dos picos de PIO foi significativamente maior na CTD $(20,30 \pm 1,03 \mathrm{mmHg})$ do que no TSH $(17,37 \pm 0,78 \mathrm{mmHg}), \quad p<0,001$ (Tabela 1).

A média dos valores de flutuação também foi maior na CTD $(10,17 \pm$ $0,90)$ do que no TSH $(5,0 \pm 0,45), p<0,001$ (Tabela 1).

O valor da flutuação em percentil na CTD foi de $90,46 \%,(70,00 ; 129,90)$ enquanto que no TSH foi de 35,42\%, (27,92;56,95), $p<0,001$ (Tabela 1).

Tabela 1: Valores de média (M) e desvio padrão (s) de pressão intra-ocular $(\mathrm{mmHg})$ de picos e flutuação e valores de mediana (Md) e quartís (Q2) da flutuação em percentil, na curva tensional diária(CTD) e teste de sobrecarga hídrica (TSH)

\begin{tabular}{cccc}
\hline & CTD & TSH & $\boldsymbol{p}$ \\
\hline Pico & $20,30 \pm 1,03$ & $17,37 \pm 0,78$ & $<0,001^{(1)}$ \\
Flutuação (M) & $10,17 \pm 0,90$ & $5,0 \pm 0,45$ & $<0,001^{(1)}$ \\
Flutuação em \% & $90,46(70,00 ; 129,90)$ & $35,42(27,92 ; 56,95)$ & $<0,001^{(2)}$ \\
\hline
\end{tabular}

(1) Teste t de Student para amostras dependentes.

(2) Teste de Wilcoxon para amostras dependentes. 
A PIO das $6 \mathrm{~h}$ da manhã mostrou diferença estatisticamente significativa quando realizada com o paciente deitado $(17,47 \mathrm{mmHg})$ e sentado $(14,30$ $\mathrm{mmHg})$, sendo significativamente maior com o paciente deitado. $(p<0,001)$ (Tabela 2).

Tabela 2: Valores de média (M) e desvio padrão (s) de pressão intra-ocular ( $\mathrm{mmHg}$ ) obtida às 6 horas da manhã com o paciente deitado e sentado

\begin{tabular}{cccc}
\hline & \multicolumn{3}{c}{$\mathbf{6 h}$} \\
\cline { 2 - 3 } $\boldsymbol{P I O}$ & Deitado & Sentado & $\boldsymbol{P}^{(1)}$ \\
\hline & $17,47 \pm 1,10$ & $14,30 \pm 0,57$ & $<0,001$ \\
\hline
\end{tabular}

(1)Teste t de Student para amostras dependentes.

A maioria dos picos na CTD ocorreu às 3h (40\%), seguida pelo horário das $6 \mathrm{~h}$, deitado $(25,71 \%)$ (Tabela 3).

Tabela 3: Distribuição dos picos por horário, em freqüência absoluta (número de vezes em que ocorreram) e em percentual

\begin{tabular}{ccc}
\hline Horário & Freqüência absoluta & Percentual relativo \\
\hline $9 \mathrm{~h}$ & 2 & $5,71 \%$ \\
$12 \mathrm{~h}$ & 4 & $11,43 \%$ \\
$15 \mathrm{~h}$ & 0 & $0,00 \%$ \\
$18 \mathrm{~h}$ & 1 & $2,86 \%$ \\
$21 \mathrm{~h}$ & 0 & $0,00 \%$ \\
$24 \mathrm{~h}$ & 5 & $14,29 \%$ \\
3h & 14 & $40,00 \%$ \\
$6 \mathrm{~h}$ deitado & 9 & $25,71 \%$ \\
$6 \mathrm{~h}$ sentado & 0 & $0,00 \%$ \\
\hline Total & $35^{*}$ & $\mathbf{1 0 0 , 0 0 \%}$
\end{tabular}

( ) A freqüência de 35 ocorreu em função do pico ter ocorrido em mais de um horário. 
Excluindo a medida das 3 horas da manhã, a maioria dos picos na CTD ocorreu às $6 \mathrm{~h}$ deitado $(45,5 \%)$, seguida pelo horário das $12 \mathrm{~h}(15,15 \%)$ (Tabela 4).

Tabela 4: Distribuição dos picos por horário, em freqüência absoluta (número de vezes em que ocorreram), sem as 3 h da manhã (número de vezes em que ocorreram) e em percentual.

\begin{tabular}{ccc}
\hline Horário & Freqüência absoluta & Percentual relativo \\
\hline $9 \mathrm{~h}$ & 2 & $6,06 \%$ \\
$12 \mathrm{~h}$ & 5 & $15,15 \%$ \\
$15 \mathrm{~h}$ & 1 & $3,03 \%$ \\
$18 \mathrm{~h}$ & 2 & $6,06 \%$ \\
$21 \mathrm{~h}$ & 1 & $3,03 \%$ \\
$24 \mathrm{~h}$ & 5 & $15,15 \%$ \\
$6 \mathrm{~h}$ deitado & 15 & $45,45 \%$ \\
6h sentado & 2 & $6,06 \%$ \\
\hline Total & $3^{*}$ & $100,00 \%$ \\
\hline
\end{tabular}

( ) A freqüência de 33 ocorreu em função do pico ter ocorrido em mais de um horário.

Excluindo a medida das 6 horas da manhã deitado, a maioria dos picos na CTD ocorreu às 3 da manhã (50\%), seguida pelo horário das 24 horas $(15,6 \%)$ (Tabela 5 ). 
Tabela 5: Distribuição dos picos por horário, em freqüência absoluta (número de vezes em que ocorreram), sem as $6 \mathrm{~h}$ deitado e em percentual.

\begin{tabular}{ccc}
\hline Horário & Freqüência absoluta & Percentual relativo \\
\hline 9h & 4 & $12,5 \%$ \\
$12 \mathrm{~h}$ & 4 & $12,5 \%$ \\
$15 \mathrm{~h}$ & 0 & $0,0 \%$ \\
$18 \mathrm{~h}$ & 1 & $3,1 \%$ \\
$21 \mathrm{~h}$ & 0 & $0,0 \%$ \\
$24 \mathrm{~h}$ & 5 & $15,6 \%$ \\
3h & 16 & $50,0 \%$ \\
6h sentado & 2 & $6,3 \%$ \\
\hline Total & $\mathbf{3 2 *}$ & $100,0 \%$
\end{tabular}

() A freqüência de 32 ocorreu em função do pico ter ocorrido em mais de um horário.

Excluindo a medida das 3 horas da manhã e 6 horas deitado, a maioria dos picos na CTD ocorreu às $6 \mathrm{~h}$ sentados $(25,7 \%)$, seguida pelo horário das $12 \mathrm{~h}$ e 24 horas (20\%) (Tabela 6).

Tabela 6: Distribuição dos picos por horário, em freqüência absoluta (número de vezes em que ocorreram), sem as medidas das $3 \mathrm{~h}$ e $6 \mathrm{~h}$ (deitado) da manhã e em percentual.

\begin{tabular}{ccc}
\hline Horário & Freqüência absoluta & Percentual relativo \\
\hline $9 \mathrm{~h}$ & 5 & $14,3 \%$ \\
$12 \mathrm{~h}$ & 7 & $20,0 \%$ \\
$15 \mathrm{~h}$ & 3 & $8,6 \%$ \\
$18 \mathrm{~h}$ & 2 & $5,7 \%$ \\
$21 \mathrm{~h}$ & 1 & $2,9 \%$ \\
$24 \mathrm{~h}$ & 7 & $20,0 \%$ \\
$6 \mathrm{~h}$ sentado & 9 & $25,7 \%$ \\
\hline Total & $35^{*}$ & $100,0 \%$
\end{tabular}

( ) A freqüência de 35 ocorreu em função do pico ter ocorrido em mais de um horário. 
A maioria dos picos no TSH ocorreu no momento $45(26,30 \%)$, seguida pelo horário das 6 h, deitado $(25,71 \%)$ (Tabela 3 e Figura 1).

Tabela 7: Distribuição dos picos por horário, em freqüência absoluta (número de vezes em que ocorreram), dos momentos do TSH e em percentual.

\begin{tabular}{ccc}
\hline Horário & Freqüência absoluta & Percentual relativo \\
\hline M 0 & 5 & $13,2 \%$ \\
M15 & 9 & $23,7 \%$ \\
M 30 & 6 & $15,8 \%$ \\
M 45 & 10 & $26,3 \%$ \\
M 60 & 8 & $21,1 \%$ \\
\hline Total & 38 & $100 \%$ \\
\hline
\end{tabular}

M 0- momento zero- início do teste

M15- (momento 15)- 15 minutos após a ingestão da água

M30-( momento 30)- 30 minutos após a ingestão da água

M45-( momento 45)- 45 minutos após a ingestão da água

M60-( momento 60)- 60 minutos após a ingestão da água

\section{DISCUSSÃO}

A ocorrência de pico e flutuação da PIO tem sido associada à progressão de defeitos de campo visual em pacientes glaucomatosos ${ }^{(6,7,12-}$ 15,18,22,35,46,48. A PIO é o mais importante fator de risco para desenvolvimento e progressão da doença $a^{6-8,49}$ e o único que pode ser tratado ${ }^{9-11}$.

A PIO sofre variações ao longo do dia e os autores divergem quanto aos horários em que ocorrem os picos: pela manhã ${ }^{23,27,50}$, ou no período da noite $31,51-53$ 
A avaliação de pico e flutuação da PIO em pacientes com glaucoma deveria ser feita durante as 24 horas. Na prática, esta avaliação é feita através da CTD, porém a realização do exame é difícil. Como alternativa para detectar pico e flutuação de PIO, tem sido utilizado o TSH ${ }^{11,37-39}$.

No presente estudo, a comparação da média dos valores de pico de PIO da CTD (medidas ao longo das 24 horas) com o TSH mostrou diferença significativa entre ambos os testes, com valores de $20,30 \mathrm{mmHg}$ e 17,37 $\mathrm{mmHg}$, respectivamente, na CTD e TSH. Hu et al ${ }^{44}$ que também compararam pico de PIO de avaliação de 24 horas, feita em laboratório do sono, com os do TSH também encontraram valor maior na CTD $(22,4 \mathrm{mmHg})$ do que no TSH $(19,9 \mathrm{mmHg})$. O estudo de $\mathrm{Hu}$ et $\mathrm{al}^{44}$ também foi feito em pacientes portadores de GPAA tratados, mas incluía apenas 11 pacientes.

Meirelles et $a{ }^{45}$ estudaram pico da PIO da CTD (7 medidas ao longo das 24 horas) e comparam com as do TSH em pacientes com GPAA sem tratamento, e encontraram valores mais elevados no $\mathrm{TSH}(26,84 \mathrm{mmHg})$ do que na CTD $(25,77)$. Os valores mais altos de pico tanto na CTD como no $\mathrm{TSH}$, observados por Meirelles et $\mathrm{al}^{45}$, poderiam ser explicados pelo fato dos pacientes não estarem em uso de nenhuma medicação hipotensora. Da mesma forma, no estudo de $\mathrm{Helal}^{39}$, com 11 pacientes, a média dos picos de PIO foi de $26,32 \mathrm{mmHg}$ na CTD e $25,64 \mathrm{mmHg}$ no TSH, e os valores também são elevados, pois os pacientes também não estavam em uso de medicação. Já os picos mais altos no TSH, poderiam ser explicados pois não foi realizada 
a medida das 3 horas da manhã, no leito, que no presente estudo, foi responsável por $40 \%$ dos picos de PIO.

No presente estudo, a flutuação de PIO na CTD foi significantemente maior do que no TSH, com valores de $10,17 \mathrm{mmHg}$ na CTD e 5,0 $\mathrm{mmHg}$ no TSH, Em porcentagem, a flutuação foi de $90,46 \%$ no CTD e 35,42 \% no TSH. Meirelles et al $^{45}$ encontraram flutuação de $8 \mathrm{mmHg}$ na associação (curva ambulatorial e TSH) e na CTD: $9,6 \mathrm{mmHg}$, que apesar de valores mais próximos também foram significativamente diferentes. No trabalho de Helal ${ }^{39}$ que não estudou flutuação e sim a variação entre: pico de PIO menos a PIO basal, os valores dessa "flutuação" de PIO foram semelhantes de $5,63 \mathrm{mmHg}$ e 5,54 na CTD e TSH, respectivamente, portanto contraditórios em relação aos anteriores. No estudo de $\mathrm{Hu}$ et $\mathrm{al}^{44}$ que não cita valores, os autores afirmam que não houve correlação no valor da flutuação realizada no laboratório do sono.

Os valores da flutuação na minicurva geralmente são bastante inferiores aos observados nos estudos com a CTD (curva completa), provavelmente pois são realizadas medidas apenas nos horários habituais de consultório, onde os valores de PIO são menores ${ }^{35,37,50,54}$. Kumar et $\mathrm{al}^{37}$, encontraram média de flutuação de 2,3mmHg na minicurva e de $2,2 \mathrm{mmHg}$ no $\mathrm{TSH}$.

Foi observado neste estudo queda da média da PIO imediatamente ao levantar: a PIO das 6 horas foi de $17,47 \mathrm{mmHg}$ (deitado) e 14,30 $\mathrm{mmHg}$ (sentado). Zeimer et al $^{55}$ afirmam que a PIO tem rápido declínio pela manhã, onde pode cair de 2 a $18 \mathrm{mmHg}$ até 1 hora após levantar. 
Nos estudos que realizaram a minicurva, ou CTD incompleta os horários de pico foram contraditórios: 17 horas $^{26}, 24$ horas $^{17}$ Isso poderia ser explicado pelo fato do verdadeiro pico ocorrer na maioria dos pacientes durante a madrugada, em posição supina. Na literatura os poucos estudos que avaliaram a PIO de 24 horas também observaram pico durante a madrugada ${ }^{31}$ : 1:30 hora $^{56}, 2$ horas $^{36}, 3: 35 h^{53}$ e início da manhã: 5:30h ${ }^{52}$ e 6 horas no leito ${ }^{27,35,45}$.

Excluindo a medida das 3 horas da manhã, a maioria dos picos na CTD neste estudo, passou a ocorrer às 6 h deitado (45,5\%), freqüência próxima a encontrada por Rodrigues et $\mathrm{al}^{27}$ que observaram ocorrência de picos de PIO em $41,67 \%$ na medida das 6 horas (paciente deitado), em pacientes glaucomatosos ou suspeitos de glaucoma. Da mesma forma, Liu et $\mathrm{al}^{57}$ ao estudarem a variação da PIO em 24 horas, em indivíduos saudáveis, observaram que houve aumento noturno da PIO e diminuição da PIO no período diurno. Sendo assim, as medidas das 3 horas e 6 horas no leito têm extrema importância na detecção de picos e flutuações de PIO.

Ao excluir a medida das 6 horas da manhã deitado, aumentou ainda mais a freqüência dos picos às 3 da manhã (50\%), seguida pelo horário das 24 horas, com apenas $15,6 \%$. Liu et al $^{58}$ sugerem, ao estudar em população idosa sem glaucoma, que ocorra elevação da PIO com a mudança da posição vertical para supina, que se inicia aproximadamente às 23:30 horas e mantem-se elevada durante toda a madrugada até o início da manhã.

Excluindo as medidas das 3 horas da manhã e 6 horas deitado, a maioria dos picos $(25,7 \%)$ na CTD ocorreu às $6 \mathrm{~h}$ (sentado). Barkana et $\mathrm{al}^{35}$ ao 
estudarem pacientes glaucomatosos em uso de medicação hipotensora ocular, observaram pico de $\mathrm{PIO}$ às 6 horas no leito, em 7 dos 22 pacientes, e concluíram também que $69 \%$ dos picos de PIO ocorrem fora do horário de consultório. Da mesma forma Moased et al $^{52}$ também observaram que a posição supina noturna detectou mais picos pressóricos.

Embora o TSH possa contribuir para avaliação de pico e flutuação de PIO nos pacientes glaucomatosos e hipertensos oculares, hipoestima os valores de ambos. Assim, novos estudos devem continuar na busca de outros métodos de avaliação da PIO durante as 24 horas do dia mais práticos do que a CTD.

\section{CONCLUSÕES}

O TSH subestima pico e flutuação da PIO em pacientes portadores de GPAA e HO. A maior incidência de pico de PIO ocorreu às 3 horas da manhã (40\%). Houve influencia postural na avaliação das 6 horas da manhã. O melhor horário para detecção de pico de PIO é em ordem decrescente: 3 horas da manhã, 6 horas da manhã deitado e 6 horas da manhã, sentado. 


\section{REFERÊNCIAS BIBLIOGRÁFICAS}

1. Resnikoff S, Pascolini D, Etya'ale D, Kocur I, Pararajasegaram R, Pokharel GP, et al. Global data on visual impairment in the year 2002. Bull World Health Organ. 2004;82(11):844-51.

2. Quigley HA, Broman AT. The number of people with glaucoma worldwide in 2010 and 2020. Br J Ophthalmol. 2006;90:262-7.

3. Shields MB. Epidemiologia do glaucoma In: Shields MB. Tratado de Glaucoma. 5 ed. Rio de Janeiro: Cultura médica; 1998. p.191-2.

4. Mello PAAM, Mandia Jr C. $2^{\circ}$ Consenso Brasileiro de Glaucoma Primário de Ângulo Aberto. São Paulo: Sociedade Brasileira de Glaucoma; 2005.p.9.

5. Sommer A, Tielsch JM, Katz J, Quigley HA, Gottsch JD, Javitt J, Singh K. Relationship between intraocular pressure and primary open angle glaucoma among white and black americans. The Baltimore Eye Survey. Arch Ophthalmol. 1991;109:1090-5.

6. Bergea B, Bodin L, Svedbergh B. Impact of intraocular pressure regulation on visual fields in open angle- glaucoma. Ophthalmology. 1999;106:997-1004. 
7. AGIS Investigators. The Advanced Glaucoma Intervention Study (AGIS): 7. The relationship between control of intraocular pressure and visual field deterioration. Am J Ophthalmol. 2000;130:429-40.

8. Sommer A. Intraocular pressure and glaucoma. Am J Ophthalmol. 1989;107:186-8.

9. Mao LK, Stewart WC, Shields B. Correlation between intraocular pressure control and progressive glaucomatous damage in primary open- angle glaucoma. Am J Ophthalmol. 1991;111:51-5.

10. Palmberg P. What is about pressure that really matthers in glaucoma? Ophthalmology. 2007;114:203-4.

11. Malerbi FK, Hatanaka M, Vessani RM, Susanna R Jr. Intraocular pressure variability in patients who reached target intraocular pressure. Br J Ophthalmol. 2005;89:540-2.

12. Leske MC, Hussein M E,, Hussein M, Bengtsson B, Hyman L, Komaroff et al. Factors for glaucoma progression and the effect of treatment: the Early Manifest Glaucoma Trial. Arch Ophthalmol.2003;121: 28-56.

13. Heijl A, Leske MC, Bengtsson B, Hyman L, Bengtsson B, Hyman L, et al. Reduction of intraocular pressure and glaucoma progression: results from the Early Manifest Glaucoma Trial. Arch Ophthalmol. 2002; 120:1268-79. 
14. European Glaucoma Prevention Study (EGPS) Group. Predictive factors for open -angle glaucoma among pacients with ocular hypertension in the European Glaucoma Prevention Study. Ophthalmology. 2007;114:3-9.

15. Collaborative Normal-tension glaucoma study group. The effectiveness of intraocular pressure reduction in the treatment of normal-tension glaucoma. Am J OphthImol. 1998;126:498-505.

16. O'Brien C, Schartz B, Takamoto T, Wu DC. Intraocular pressure and the rate of visual field loss in chronic open-angle glaucoma. Am J Ophthlamol. 1991;111:491-500.

17. Jonas JB, Budde W, Stroux A, Oberacher-Velten IM, Junemann A. Single intraocular pressure measurement and diurnal intraocular pressure profiles. Am J Ophthalmol. 2005;139:1136-7.

18. Medeiros FA, Weinreb RN, Zangwill LM, Alencar LM, Sample PA, Vasile C, et al. Long-term intraocular pressure fluctuations and risk of conversion from ocular hypertension to glaucoma. Ophthalmology. 2008;115:934-40.

19. Gordon MO, Beiser JA, Brandt JD, Heuer DK, Higginbotham EJ, Johnson CA, et al. The ocular hypertension treatment study. Baseline factors that predict the onset of primary open-angle glaucoma. Arch Ophthalmol. 2002;120:714-20. 
20. Asrani S, Zeimer R, Wilensky J, Gieser D, Vitale S, Lienmuth K. Large diurnal fluctuations in intraocular pressure are an independent risk factor in patients with glaucoma. J Glaucoma. 2000;9:134-42.

21. Caprioli J, Coleman AL. Intraocular pressure fluctuation. Ophthalmology. 2008;115:1123-9.

22. Nouri- Mahdavi K, Hoffman BA, Coleman AL, Liu G, Li G, Gaasterland D, Caprioli J. Peditctive Factors for glaucomatous visual field progression in the Advanced Glaucoma Intervention Study. Ophthalmology. 2004; $111: 1627-35$.

23. Sacca SC, Rolando M, Marletta A, Macrí A, Cerqueti P, Ciurlo G. Fluctuations of intraocular pressure during the day in open-angle glaucoma, normal tension glaucoma and normal subjects. Ophthalmologica. 1998;212:115-9.

24. Singh K, Shrivastava A. Intraocular pressure fluctuations: how much do they matter? Curr Opin Ophthalmol. 2009;20:84-7.

25. Bengtsson B, Leske MC, Hyman L, Heijil A. Fluctuation of intraocular pressure and glaucoma progression in the early manifest glaucoma trial. 2007. Ophthalmology. 2007:144:205-9. 
26. Rota-Bartelink AM, Pitt A, Story I. Influence of diurnal variation on the intraocular pressure measurement of treated primary open-angle glaucoma during office hours. J Glaucoma. 1996;5:410-15.

27. Rodrigues LD, Silva, M R B M, Schellini SA, Jorge, EN. Picos de pressão intra-ocular: comparação entre curva tensional diária, minicurva e medida da pressão intra-ocular às 6 horas. Arq Bras Oftalmol. 2004; 67:127-31.

28. Hasekawa K, Ishida K, Sawada A, Kawase K, Yamamoto T. Diurnal variation of intraocular pressure in suspect normal- tension glaucoma. Jpn J Ophthalmol. 2006;50:449-54.

29. Hughes E, Spry P, Diamond J. 24-Hour monitoring of intraocular pressure in glaucoma management: a retrospective review. J Glaucoma. 2003; $12: 232-6$.

30. Drance SM. Diurnal variation of intraocular pressure in treated glaucoma: significance in patients with chronic simple glaucoma. Arch Ophthalmol.1963; 70:302-11.

31. Romanet J-P, Maurent-Palombi K, Noël C, Bourdon L, Pépin J-L, Moullon M, Buguet A. Variations nycthémerales de la pression intraoculaire. J Fr Ophtalmol. 2004;27:2519-26.

32. Pointer JS. Human intraocular pressure and its diurnal variation in healthy subjects. Ophthalmic Physiol Opt 1999; suppl 2: S43-S48 
33. Shields MB. A pressão intraocular In: Shields MB. Glaucoma. 2 ed. São Paulo: Panamericana; 1989. p.47-73.

34. Waisberg Y. Medida das seis horas da manhã, da curva diária de pressão intra-ocular: quando ela é necessária? Rev Bras Oftalmol. 1982;41:34-50.

35. Barkana Y, Anis S, Liebmann J, Tello C, Ritch R. Clinical Utility of intraocular pressure monitoring outside of normal office hours in patient with glaucoma. Arch Opthalmol. 2006;124:793- 7.

36. Konstas AGP, Mantziris DA, Cate EA, Stewart WC. Effect of timolol on the diurnal intraocular pressure in exfoliation and primary open angle glaucoma. Arch Ophthalmol. 1997;115:975-9.

37. Kumar RS, Guzman HP, MS Sung, Ong PY, Goldberg I. Does peak intraocular pressure by water drinking test reflect peak circadian levels? A pilot study. Clin Exp Ophthalmol. 2008:36:312-5.

38. Medeiros FA, Leite CA, Susanna Jr R. Correlação entre os picos pressóricos da curva tensional diária e o teste de sobrecarga hídrica. Rev Bras Oftalmol. 2001;60:418-23.

39. Helal Jr J. Contribuição ao estudo da pressão intra-ocular: picos de pressão intra-ocular na curva diária de pressão e na prova de sobrecarga hídrica. Rev Bras Oftalmol. 1988;47:7-12. 
40. Susanna R Jr, Vessani RM, Sakata L, Zacarias LC, Hatanaka M. The relation between intraocular pressure peak in the water drinking test and visual field progression in glaucoma. Br J Ophthalmol. 2005;89:1298-301.

41. Frota ACA, Meirelles SHS, Ávila E, Yamane Y. Correlação entre picos pressóricos de teste de sobrecarga hídrica e curva ambulatorial de presssão intra-ocular em glaucoma primário de ângulo aberto suspeito. Rev Bras Oftalmol. 2005; 64:383-7.

42. Vasconcelos- Moraes CG, Susanna Jr R. Correlation between water drinking test and modified diurnal tension curve in untreated glaucomatous eyes. Clinics. 2008;63:433-6.

43. Miller $\mathrm{D}$. The relationship between diurnal tension variation and the waterdrinking test. Am J Ophthlamol. 1964;58:243-6.

44. Hu WD, Medeiros FA, Alencar LMP, Liu JH, Susanna R, Weinreb RN.The correlation between the water drinking test and 24 hour intraocular pressure measurements in glaucomatous eyes. Poster presented during the Meeting of the Association for Research in Vision and Ophthalmology (ARVO) in May 2007, Fort Lauderdale-FL.

45. Meirelles SHS, Yamane R, Alvares RM, Botelho PB, Morais FB, Moreira PB, et al. Estudo comparativo entre a curva tensional diária de pressão intra-ocular e a associação da curva ambulatorial com o teste de sobrecarga hídrica no glaucoma primário de ângulo aberto, glaucoma de pressão normal e olhos normais. Arq Bras Oftalmol. 2007;70:471-9. 
46. Dinn RB, Zimmerman B, Shuba LM, Doan AP, Maley MK, Greenlee EC, et al. Concordance of diurnal intraocular pressure between fellow eyes in primary open-angle glaucoma. Ophthalmology. 2007; 114:915-20.

47. Lichter PR, Musch DC, Gillespie BW, Guire KE, Janz NK, Wren PA, et al. Interin clinical outcomes in the collaborative initial glaucoma treatment Study Comparing initial treatment randomized to medications or Surgery. Ophthalmology. 2001;108:1943-53.

48. Susanna R Jr, Weinreb RN. Glaucoma: perguntas e respostas. Rio de Janeiro: Cultura Médica;2005.

49. Sommer A, Intraocular pressure and glaucoma. Am J Ophthalmol. 1989:107:186-8.

50. David R, Zangwill L, Briscoe D, Dagan M, Yagev R, Yassur Y. Diurnal introvular pressure variations: an analysis of 690 diurnal curves. $\mathrm{Br} \mathrm{J}$ Ophthalmol. 1992;76:280-3.

51. Liu JHK, Kripke DF, Hoffman RE, TA MD, Loving RT, Rex KM, et al. Nocturnal elevation of intraocular pressure in young adults. Invest Ophthalmol Vis Sci . 1998;39:2707-11.

52. Mosaed S, Liu JHK, Weinreb RN. Correlation between office and peak nocturnal intraocular pressure in healthy subjects and glaucoma pacients. Am J Ophthlmol. 2005;139:320-4. 
53. Buguet A, Romanet JP. 24- hour (nyctohemeral) and sleep-related variations of intraocular pressure in healthy white individuals. Am J Ophthalmol. 1994;117:342-7.

54. Susanna R Jr, Nicoleta MT, Oga E. Efeito aditivo na redução da pressão intra-ocular do latanoprost a associção de dorzolamida e timolol. Rev Bras Oftalmol. 1998.57:129-34.

55. Zeimer RC, Wilensky J T, Gieser DK. Presence and rapid decline of early morning intraocular pressure peaks in glaucoma pacients. Ophthalmology 1990;97:547-50.

56. Deokule SP, Doshi A, Vizzeri G, Medeiros FA, Liu JHK, Bowd C, et al. Relahionship of the 24-hour pattern of intraocular pressure with optic disc appearance in primary open-angle glaucoma. Ophthalmology. 2009;116:833-9.

57. Liu JHH, Sit AJ, Weinre RN. Variation of 24-hour intraocular pressure in healthy individuals. Ophthalmology. 2005;112:1670-5.

58. Liu JHK, Kripke DF, Twa MD, Hoffman RE, Masnberger SL, Rex KM, et al. Twenty-four-hour pattern of intraocular pressure in the aging population. Invest Ophthalmol Vis Sci. 1999; 40: 2912-7. 


\section{Capítulo II}

Comparação de pico e flutuação de PIO entre

2 TSH realizados em horarios diferentes 


\section{Comparação de pico e flutuação de PIO entre 2 TSH realizados em horários diferentes}

\section{RESUMO}

OBJETIVOS: Comparar pico e flutuação de pressão intra-ocular (PIO) entre dois testes de sobrecarga hídrica (TSH) realizados em períodos diferentes do dia. Correlacionar os valores da espessura central da córnea (ECC) com os valores de pressão intra-ocular ao longo do TSH. MATERIAL E MÉTODOS: 29 olhos de 29 pacientes portadores de glaucoma primário de ângulo aberto ou hipertensão ocular foram submetidos a 2 TSH no mesmo dia (6:00 h e 14:00 h). Os pacientes ingeriam 1 litro de água, na temperatura ambiente em até 5 minutos. A PIO foi avaliada imediatamente antes e durante uma hora após a ingestão de água, com intervalos de 15 minutos entre as medidas. Foram avaliadas: PIO basal, pico (maior valor da PIO durante o TSH) e flutuação (maior valor da PIO menos o menor valor da PIO durante o TSH). Foram calculados valores de média (M) e desvio padrão (s) para PIO basal e pico e mediana (Md) e quartís (Q2) para: flutuação, espessura central da córnea e os valores de PIO em diversos momentos dos 2 TSH. Para flutuação também foi calculada a porcentagem. Para comparação de médias foi realizado o teste $\mathrm{t}$ de Student para amostras dependentes. Foi utilizado teste de Wilcoxon para comparação de medianas entre amostras dependentes. Foi considerado nível de significância $p<0,05$. RESULTADOS: No TSH realizado às 6 horas da manhã a média dos valores de PIO basal foi de $14,31 \pm 3,38$ $\mathrm{mmHg}$ e no $\mathrm{TSH}$ realizado às 14 horas foi de $12,72 \pm 3,5 \mathrm{mmHg}$, sendo a 
diferença estatisticamente significativa $(p=0,022)$. A média dos valores do pico de PIO foi semelhante nos dois TSH, 17,34 $\pm 4,52 \mathrm{mmHg}$ e de $16,79 \pm 3,76$ $\mathrm{mmHg}$, respectivamente no TSH das 6 e das 14 horas $(p=0,431)$. A mediana da flutuação foi de $5,00 \mathrm{mmHg}$ em ambos TSH $(p=0,385)$. No entanto os quartís foram diferentes, no $\mathrm{TSH}$ das $6 \mathrm{~h}, 75 \%$ dos pacientes apresentaram valores de flutuação acima de 6 mmHg e no TSH das 14h, 75\% apresentaram valores acima de 7,5 mmHg. Em porcentagem, a mediana da flutuação foi de 33,33\% no TSH das 6 horas da manhã, e de 40,00\%, no TSH das 14 horas, porém, com a diferença não significativa $(p=0,127)$. A mediana da ECC foi de $574,0(551,0 ; 602,5) \mu \mathrm{m}$ e $545,0(523,2 ; 569,0) \mu \mathrm{m}$, respectivamente às 6 e 14 horas, $p<0,001$. A ECC decresceu ao longo do teste das 6 horas, sendo $o$ decréscimo estatisticamente significativo nos momentos 30 e 60 . No teste das 14 horas a ECC manteve-se estável ao longo do teste. CONCLUSÕES: Pico e flutuação da PIO, no TSH da manhã e da tarde apresentaram valores semelhantes. Conclui-se assim que o TSH pode ser realizado em qualquer horário do dia. A elevação da pressão intra-ocular do TSH não está relacionada com alterações de ECC.

PALAVRAS-CHAVE: glaucoma primário de ângulo aberto + hipertensão ocular+ teste de sobrecarga hídrica + pico de pressão intra-ocular+ flutuação da pressão intra-ocular 


\section{Comparison IOP peak and fluctuation realized in two WDT, in different times of the day.}

\section{ABSTRACT}

PURPOUSE: Compare peak and fluctuation of intraocular pressure (IOP) between two water drinking tests (WDT) were done at different times of day. Correlate the values of central corneal thickness (CCT) with the values of IOP at the WDT. METHODS: 29 eyes of 29 glaucoma patients with open angle glaucoma or ocular hypertension were submitted to 2 WDT (6AM and 2 PM) on the same day. The WDT involved ingestion of 1 liter of tap water in 5 minutes. IOP was measured immediately before and one hour after ingestion of water at intervals of 15 minutes between measurements The parameters available were: baseline IOP, peak (highest value of IOP during WDT) and fluctuation (difference between highest and lowest value of IOP during WDT). Were calculated mean values (M) and standard deviation (s) for basal and peak IOP and median (Md) and quartiles (Q2) to: fluctuations, CCT and IOP values at different times of 2 WDT. For fluctuation was also calculated the percentage. For comparison of means was performed Student's $t$ test for dependent samples. Wilcoxon test was used to compare medians between dependent samples. The level of significance was considered $p<0.05$. RESULTS: In WDT performed at 6AM the mean baseline IOP was $14.31 \pm 3.38 \mathrm{mmHg}$ and in the WDT performed at 2PM was $12.72 \pm 3.5 \mathrm{mmHg}$, with no significant difference $(p=0.022)$. The mean peak IOP was similar in both WDT, $17.34 \pm 4.52 \mathrm{mmHg}$ 
and $16.79 \pm 3.76 \mathrm{mmHg}$, respectively at $6 \mathrm{AM}$ and $2 \mathrm{PM}(\mathrm{p}=0.431)$. The median fluctuation was $5.00 \mathrm{mmHg}$ in both WDT ( $p=0.385)$. However, the quartiles were different, the WDT at $6 \mathrm{AM}, 75 \%$ of patients with values fluctuating above and $6 \mathrm{mmHg}$ in the 2PM WDT, 75\% had values above 7.5 $\mathrm{mmHg}$. In percentages, the median fluctuation was $33.33 \%$ in the WDT of 6 AM, and $40.00 \%$ for WDT of $2 \mathrm{PM}$, but with no significant difference $(p=$ 0.127). The median CCT was $574.0(551.0,602.5) \mu \mathrm{m}$ and $545.0(523.2,569.0)$ $\mu \mathrm{m}$ respectively at $6 \mathrm{AM}$ and $2 \mathrm{PM}, \mathrm{p}<0.001$. ECC decreased over the test of $6 \mathrm{AM}$, and the statistically significant decrease 30 and 60 minutes after drinking water. In the WDT at 2PM the ECC remained stable. CONCLUSION: IOP peaks and IOP fluctuation, in both (morning and afternoon) WDT, had the same values. The WDT could be done anytime of the day. There's no relation between IOP elevation and CCT. In the WDT at 2PM the ECC remained stable.

KEYWORDS: open angle glaucoma+ ocular hypertension+ water drinking test+ IOP peak + IOP fluctuation. 


\section{INTRODUÇÃO}

O glaucoma é apontado como a primeira causa de cegueira irreversível no mundo, representa um dos maiores problemas oftalmológicos do planeta e estima-se que para o ano de 2010 haja 60,5 milhões de portadores da doença, sendo 4,5 milhões cegos bilateralmente ${ }^{1}$. Até o presente momento, não existe nenhum tratamento que recupere a visão perdida pelo glaucoma. No entanto a cegueira pode ser evitada, em quase todos os casos e depende de detecção precoce e tratamento adequado ${ }^{2}$.

De todas as formas de glaucoma, o glaucoma primário de ângulo aberto (GPAA) é a mais prevalente, representando $74 \%$ dos casos. O GPAA é uma neuropatia óptica crônica, progressiva, caracterizada por alterações típicas do disco óptico e da camada de fibras nervosas da retina, com repercussões características no campo visual. É acompanhado, na maioria das vezes, de pressões intra-oculares (PIO) acima de níveis considerados estatisticamente normais ${ }^{3}$.

Apesar do GPAA ser uma doença multifatorial, o aumento da PIO é o mais importante fator de risco para o desenvolvimento e progressão da doença $^{4-8}$. Além disso, a redução da PIO é o único tratamento existente e efetivo para a doença ${ }^{9-11}$.

A importância do controle da PIO em pacientes portadores de glaucoma foi recentemente verificada em estudos multicêntricos ${ }^{6,12-16}$. Estes estudos, no entanto, divergem em relação a qual fator da PIO é mais importante para 
progressão ou conversão ${ }^{17}$ do glaucoma: PIO média ${ }^{5,6,9,12,14,18,19}$, pico ou flutuação ${ }^{20-26}$ ?

A redução da PIO média, nos estudos citados, apesar de benéfica não impediu a conversão para glaucoma ou progressão da doença em $100 \%$ dos pacientes. Isto sugere que a PIO média não foi suficientemente reduzida ou que outros fatores estariam envolvidos na conversão ou progressão, como pico de PIO e flutuação ${ }^{5,18,27}$.

A PIO varia fisiologicamente ao longo do dia e sofre influencia de fatores, tais como: variação postural, exercícios físicos, tipo de alimentação, condições sistêmicas ${ }^{2}$. A PIO também varia em pacientes glaucomatosos. Pacientes que têm a PIO controlada no horário de consultório, podem apresentar progressão de escavação do disco óptico e campo visual ${ }^{12,16,21,28}$, podendo ser causada por pico de PIO que ocorrem durante a madrugada 29,30 ou nas primeiras horas do dia ${ }^{18,28,31,32-34}$.

Outro fator, que influencia a PIO é a espessura central da córnea (ECC), e tem sido apontada como fator de desenvolvimento e progressão do glaucoma $^{14,30,35,36}$

A maneira ideal de avaliação da PIO durante as 24 horas seria por meio da curva tensional diária (CTD), onde habitualmente realizam-se 7 medidas de 3/3h, excluindo-se a medida das 3 horas da manhã, mas é difícil de ser realizada e implica na internação do paciente, mudando sua rotina diária e dificultosa para o médico.

Na procura de métodos mais práticos e simples para avaliação de pico e flutuação da PIO, tem-se realizado métodos alternativos tais como a minicurva 
e o teste de sobrecarga hídrica (TSH). A minicurva normalmente é realizada nos horários habituais de consultório, mas não detecta $60-70 \%$ picos, pois estes ocorrem na sua maioria na madrugada ou nas primeiras horas do $\mathrm{dia}^{28,37-}$ 39. O TSH tem sido usado como um método alternativo para avaliação do pico e flutuação da $\mathrm{PIO}^{40-47}$.

O TSH foi proposto recentemente para verificar como o olho recupera-se do aumento transitório da PIO. O mecanismo que explica a elevação da PIO no TSH não está claro. Uma das hipóteses seria a alteração da osmolaridade do sangue que elevaria a PIO devido à hidratação do humor vítreo. Outra teoria é que a redução da osmolaridade pode aumentar a produção do aquoso. Uma terceira hipótese seria que a diminuição da osmolaridade levaria à um estimulo do SNC endócrino, que por sua vez eleva a $\mathrm{PIO}^{48}$. Em outro estudo, autores concluem que durante o TSH há aumento transitório da $\mathrm{PIO}$, precedida por aumento da amplitude de pulso ocular e espessura coroidal, e acreditam que esse mecanismo deve explicar parte da elevação da PIO durante o teste ${ }^{49}$

Estudos compararam pico e flutuação da PIO obtidos na CTD e no TSH, e observaram correspondência ${ }^{40,41,46,50,51,56,57}$.

$\mathrm{Na}$ literatura referente ao TSH, com raras exceções ${ }^{52-55}$ os testes foram realizados no período da tarde ${ }^{11,40,51,56,57}$. Encontramos na literatura apenas um estudo $^{58}$ que realizou o TSH, nos mesmos pacientes, em mais de um horário. Não há estudo na literatura que informe qual o melhor horário de realização do TSH com objetivo de detectar pico e flutuação pela manhã quando a PIO costuma ser maior ou à tarde quando a PIO é menor. 


\section{OBJETIVOS}

1. Comparar pico e flutuação de PIO entre dois TSH realizados em períodos diferentes do dia.

2. Correlacionar os valores da espessura central da córnea (ECC) com os valores de PIO ao longo do TSH

\section{MATERIAL E MÉTODOS}

Foi feito estudo prospectivo em 29 olhos de 29 pacientes portadores de glaucoma primário de ângulo aberto ou hipertensão ocular do Ambulatório de Glaucoma do Hospital das Clínicas da Faculdade de Medicina de BotucatuUNESP. O estudo foi realizado no período de 06 de janeiro à 07 setembro de 2007. Todos os pacientes concordaram em participar do estudo, após explanação do mesmo e assinaram termo de consentimento Livre e Esclarecido. O estudo foi aprovado pelo Comitê de Ética da Faculdade de Medicina de Botucatu, sob número 2397/2007.

Todos os pacientes foram submetidos a exames de acuidade visual, refração, biomicroscopia, gonioscopia, fundoscopia e perimetria computadorizada.

Foram incluídos neste estudo os pacientes que preenchiam os seguintes critérios de inclusão: 
- Disco óptico com duas ou mais das alterações glaucomatosas: assimetria $>0,2$ entre as escavações dos dois olhos, presença de defeito em cunha, perda do padrão ISNT, vasos em baioneta, hemorragia no disco óptico e sinal de Hoyt.

- Campo visual com pelo menos: 3 pontos adjacentes não periféricos com redução $\geq 5 \mathrm{~dB}$ no gráfico pattern deviation, sendo que um deles com $\geq 10 \mathrm{~dB}$, em localização compatível com defeito glaucomatoso.

- Ausência de doença ocular ou doença neurológica que justificasse alteração de campo visual.

- $\mathrm{PIO}>$ a $21 \mathrm{mmHg}$, sem alteração de campo visual ou disco óptico. (hipertensos oculares)

- Ângulo aberto e de aspecto normal, à gonioscopia (classificação grau 3 e 4 de Shaffer).

Foram excluídos do estudo:

- Pacientes do sexo feminino com risco de gravidez,

- Pacientes que realizaram cirurgia ocular e tratamentos a laser há menos de 6 meses.

- Pacientes com acuidade visual $<20 / 40$.

- Pacientes portadores de patologia de córnea, úvea ou qualquer outra patologia que pudesse alterar a PIO.

- Pacientes portadores de Síndrome de pseudoexfoliação, com ou sem glaucoma. 
- Usuários de medicação sistêmica que pudesse interferir na PIO.

- Pacientes que não conseguissem completar todos os exames.

- Pacientes com patologia renais crônicas e insuficiência cardíaca.

A medida da acuidade visual foi feita com tabela de Snellen, com melhor correção óptica, estando o paciente a 6 metros da mesma.

A refração foi realizada com refrator automático (modelo Accuref $\mathrm{K}$, Shin- Nippon, Tókio), sem ciclopegia e foi seguida da refração dinâmica no refrator. Nos pacientes com menos de 40 anos, também foi realizada refração estática.

A gonioscopia foi realizada com a lente de Goldmann de 1 espelho, após anestesia tópica e com auxílio de metilcelulose $2 \%$.

A biomicroscopia do segmento anterior foi feita com lâmpada de fenda e foram avaliadas e descritas alterações nas pálpebras, córnea, câmara anterior, íris e cristalino.

A biomicroscopia do fundo do olho foi feita, após dilatação, com colírio de tropicamida $1 \%$ (Midriacyl $1 \%{ }^{\circledR}$ ), com auxílio de lente de Volk de 78 dioptrias. Foi realizada por 2 examinadores e as alterações foram descritas e comparadas.

O campo visual foi realizado com perímetro computadorizado Humphrey Field Analyser (modelo 740, San Leandro, Califórnia, USA), usando estratégia 24-2, e correção óptica adequada. Foi analisado por 2 examinadores. 
Para avaliar pico e flutuação de PIO foram realizados dois TSH em períodos diferentes do mesmo dia.

Os TSH foram realizados após jejum mínimo de 2 horas de sólidos e líquidos. Os pacientes ingeriam 1 litro de água, na temperatura ambiente em até 5 minutos. A PIO foi avaliada imediatamente antes e durante uma hora após a ingestão de água, com intervalos de 15 minutos entre as medidas. $\mathrm{O}$ primeiro teste de sobrecarga hídrica foi realizado às $6 \mathrm{~h}$ da manhã e o segundo à tarde, às 14 horas. Todas as medidas dos TSH foram feitas pelo mesmo examinador, que desconhecia o diagnóstico dos pacientes.

As PIO foram avaliadas com o tonômetro de Goldmann (Haag Streit, Berna, Suíça) acoplado à lâmpada de fenda, previamente calibrado. Antes da tonometria, instilou-se 1 gota de colírio preparado: $1 \mathrm{ml}$ de fluoresceína sódica 1\% (Fluoresceína sódica $1 \%{ }^{\circledR}$ ) diluído em 1 frasco de proximetacaína $0,5 \%$ $\left(\right.$ Anestalcon ${ }^{\circledR}$ )

Para avaliação da espessura central da córnea, após anestesia, utilizouse paquímetro ultrassônico (modelo PAC SCAN 300 AP Medvision- USA), em 3 momentos, antes da ingestão da água e 30 e 60 minutos após. Para cada avaliação foi considerada a média de 3 medidas consecutivas.

Considerou-se PIO basal: o valor inicial da PIO nos TSH.

Considerou-se pico: o maior valor da PIO observado durante os TSH.

Considerou-se flutuação o maior valor da PIO menos o menor valor da $\mathrm{PIO}$ nos TSH. 
Foram calculados valores de média $(\mathrm{M})$ e desvio padrão (s) para PIO basal e pico e mediana (Md) e quartís (Q2) para: flutuação, espessura central da córnea e os valores de PIO em diversos momentos dos 2 TSH. Para flutuação também foi calculada a porcentagem.

Para comparação de médias foi realizado o teste $t$ de Student para amostras dependentes. Foi utilizado teste de Wilcoxon para comparação de medianas entre amostras dependentes. Para análise, foi utilizado o programa SPSS v. 15.0. Foi considerado nível de significância $p<0,05$.

\section{RESULTADOS}

A média da idade dos 29 pacientes, era de 60,31 \pm 13.39 anos, sendo $18(62,1 \%)$ brancos e $11(37,9 \%)$ pacientes pardos, negros e amarelos. Eram 16 homens $(55,17 \%)$ e 13 mulheres $(44,83 \%)$. Dos 29 olhos analisados, 10 $(34,48 \%)$ eram portadores de hipertensão ocular, sendo 1 em tratamento e 19 $(65,52 \%)$ olhos portadores de glaucoma primário de ângulo aberto sendo que 11 estavam em uso de uma medicação e 8 estavam em tratamento com 2 ou mais hipotensores oculares.

A média dos valores de $\mathrm{PIO}$ basal no TSH realizado às 6 horas da manhã foi de $14,31 \pm 3,38 \mathrm{mmHg}$ e no TSH realizado às 14 horas foi de 12,72 $\pm 3,51 \mathrm{mmHg}$. A comparação entre esse dois valores mostrou diferença estatisticamente significativa $(p=0,022)$. A média dos valores dos picos de PIO foi semelhante nos dois TSH, 17,34 $\pm 4,52 \mathrm{mmHg}$ e de 16,79 $\pm 3,76 \mathrm{mmHg}$, respectivamente às 6 e às 14 horas, $p=0,431$ (Tabela 1 e Figura 1). 
Houve elevação da PIO em ambos TSH sendo a média dos valores de pico de PIO significativamente maior do que a média da PIO basal em ambos os TSH. No teste realizado pela manhã a diferença entre pico e PIO basal foi de 3,03 mmHg, já no teste das 14 horas, a diferença foi de 4,07 $\mathrm{mmHg}$, ambos com $\mathrm{p}<0,001$ (Tabela 1).

Tabela 1: Valores de média (M) e desvio padrão (s) de pressão intra-ocular $(\mathrm{mmHg})$ basal e pico nos 2 testes de sobrecarga hídrica.

\begin{tabular}{lccc}
\hline \multirow{2}{*}{$\boldsymbol{P I O}$} & \multicolumn{6}{c}{} & $\mathbf{1 4 h}$ & $\boldsymbol{p}$ \\
\cline { 2 - 4 } & $\boldsymbol{M} \boldsymbol{s}$ & $\boldsymbol{M} \boldsymbol{s}$ & \\
\hline Basal & $14,31 \pm 3,38$ & $12,72 \pm 3,51$ & $0,022\left(^{1}\right)$ \\
Pico & $17,34 \pm 4,52$ & $16,79 \pm 3,76$ & $0,431\left(^{1}\right)$ \\
\hline$P$ & $<0,001\left(^{1}\right)$ & $<0,001\left(^{1}\right)$ & \\
\hline
\end{tabular}

${ }^{1}$ ) Teste t de Student para amostras dependentes. Resumo descritivo em média e desvio padrão

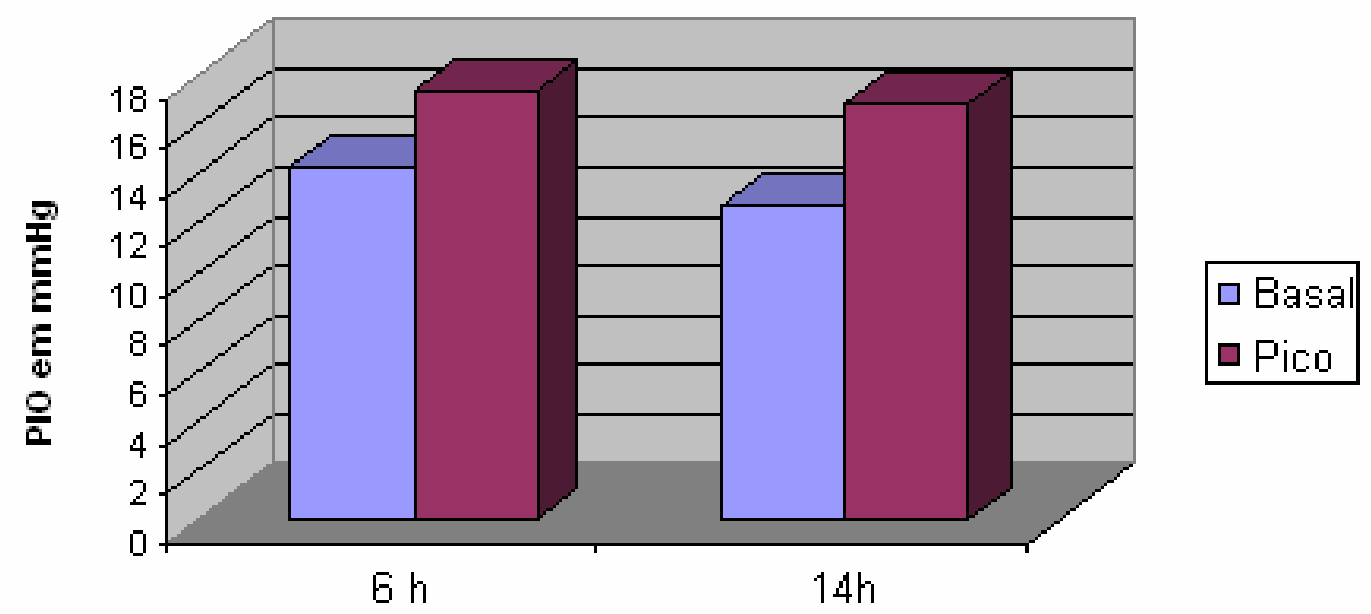

Figura 1: Valores de média $(\mathrm{M})$ de pressão intra-ocular $(\mathrm{mmHg})$ basal e pico, nos 2 testes de sobrecarga hídrica 
A mediana da flutuação em ambos TSH foi de $5,00 \mathrm{mmHg}(p=0,385)$.No entanto os quartís foram diferentes, no $\mathrm{TSH}$ das $6 \mathrm{~h}, 75 \%$ dos pacientes apresentaram valores de flutuação acima de $6 \mathrm{mmHg}$ e no TSH das $14 \mathrm{~h}, 75 \%$ apresentaram valores acima de $7,5 \mathrm{mmHg}$. Em porcentagem, a mediana da flutuação foi de 33,33\% no TSH das 6 horas da manhã, e de 40,00\%, no TSH das 14 horas, porém, com a diferença não significativa $(p=0,127)$ (Tabela 2 e Figura 2).

Tabela 2: Valores de mediana (Md) e quartís (Q2) da flutuação de PIO (mmHg e \% ) nos dois testes de sobrecarga hídrica

\begin{tabular}{|c|c|c|c|}
\hline \multirow{2}{*}{ Flutuação } & $6 h$ & $14 h$ & \multirow{2}{*}{$p$} \\
\hline & Md Q2 & Md Q2 & \\
\hline $\mathrm{mmHg}$ & $5,00(3,00 ; 6,00)$ & $5,00(3,5 ; 7,5)$ & $0,387\left(\left(^{1}\right)\right.$ \\
\hline$\%$ & $33,33(27,92 ; 56,94)$ & $40,00(30,95 ; 73,21)$ & $0,127\left(^{1}\right)$ \\
\hline
\end{tabular}

$\left({ }^{1}\right)$ Teste de Wilcoxon para amostras dependentes.

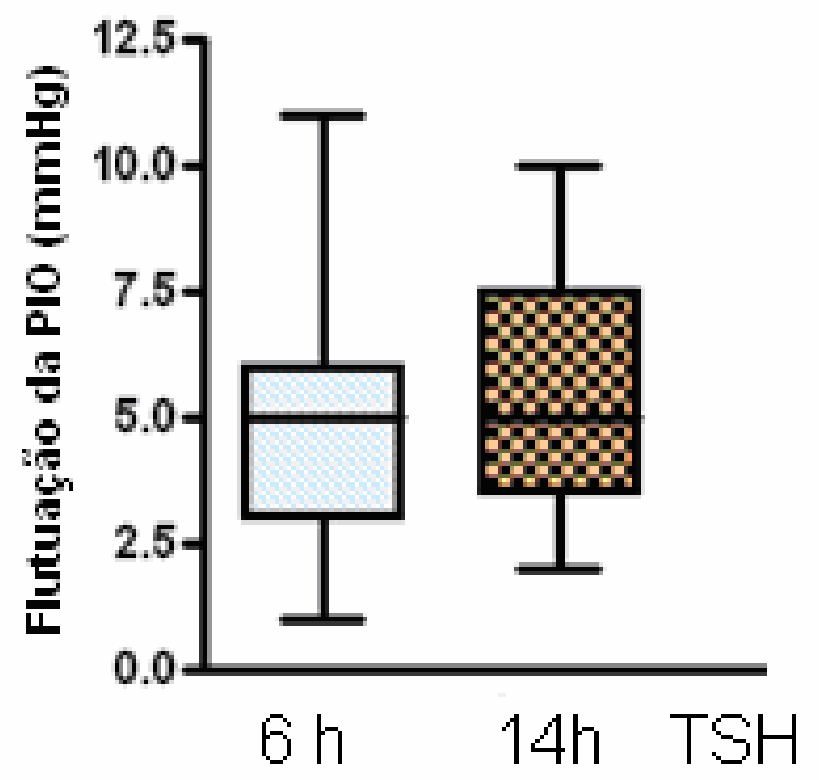


Figura 2: Valores de mediana (Md) e quartís (Q2) da flutuação de PIO $(\mathrm{mmHg})$ dos dois testes de sobrecarga hídrica

A mediana da diferença do pico de PIO menos a PIO basal foi de 2 $\mathrm{mmHg}$ no TSH das 6 horas e de $4 \mathrm{mmHg}$ no TSH das 14 horas, sendo porém esta diferença não significativa $(p=0,157)$. Em porcentagem a diferença entre pico e PIO basal foi de $14,29 \%$ no TSH das 6 horas e $28,57 \%$ no TSH das 14 horas $(p=0,125)($ tabela 3$)$.

Tabela 3: Valores de mediana (Md) e quartís (Q2) da diferença entre pico de $\mathrm{PIO}$ e PIO basal $(\mathrm{mmHg})$ nos 2 testes de sobrecarga hídrica

\begin{tabular}{cccc}
\hline $\begin{array}{c}\text { Diferença entre } \\
\text { pico de PIO } \\
\text { basal }\end{array}$ & Md Q2 & 14h & \multirow{2}{*}{$\boldsymbol{p}$} \\
\cline { 2 - 4 } & $2,00(1,00 ; 5,00)$ & $4,00(2,00 ; 5,00)$ & $0,157^{(1)}$ \\
\hline $\mathrm{mmHg}$ & $14,29(7,42 ; 36,61)$ & $28,57(14,64 ; 55,64)$ & $0,125^{(1)}$ \\
\hline$\%$ &
\end{tabular}

(1) Teste de Wilcoxon para amostras dependentes.

A tabela 4 apresenta a mediana e quartís da PIO basal e 15, 30, 45 e 60 minutos após a ingestão da água nos TSH das 6 e 14 horas. Em ambos os TSH houve aumento da PIO após a ingestão da água, sendo que o pico ocorreu no momento 30 . No teste das 14 horas, a PIO permaneceu elevada até 60 minutos após a ingestão de água.

Tabela 4: Valores de mediana (Md) e quartís (Q2) de PIO ( $\mathrm{mmHg}$ ) em todos os momentos dos 2 testes de sobrecarga hídrica

\begin{tabular}{cccccc}
\hline & M 0 & M15 & M 30 & M 45 & M 60 \\
& Md Q2 & Md Q2 & Md Q2 & Md Q2 & Md Q2 \\
\hline TSH 6h & $14,0(13,0 ; 16,5)$ & $15(12,0 ; 18,0)$ & $15,0(12,5 ; 17,5)$ & $15,0(11,5 ; 18,5)$ & $13,0(11,0 ; 18,0)$ \\
$\boldsymbol{P}^{\mathbf{1}}$ & & 0,602 & 0,043 & 0,284 & 0,858 \\
TSH 14H & $13,0(10,0 ; 14,5)$ & $13(10,5 ; 16,5)$ & $16,0(12,0 ; 16,0)$ & $15,0(13,0 ; 18,0)$ & $14,0(12,0 ; 15,5)$ \\
$\boldsymbol{P}^{\mathbf{1}}$ & & 0,343 & 0,005 & 0,001 & 0,031 \\
$\boldsymbol{P}^{\mathbf{2}}$ & 0,054 & 0,175 & 0,375 & 0,828 & 0,836 \\
\hline
\end{tabular}

(1) Comparação da PIO entre os momentos 15, 30, 45, 60 em relação a PIO basal

( ${ }^{2}$ ) Comparação da PIO, em cada momento da observação, entre os horários $6 \mathrm{~h}$ e $14 \mathrm{~h}$

M 0- momento zero- início do teste

M15- (momento 15)- 15 minutos após a ingestão da água

M30-( momento 30)- 30 minutos após a ingestão da água

M45-( momento 45)- 45 minutos após a ingestão da água

M60-( momento 60)- 60 minutos após a ingestão da água 
A espessura central de córnea (ECC) basal variou de $502 \mu \mathrm{m}$ à $665 \mu \mathrm{m}$ às 6 horas e de 488 e $647 \mu \mathrm{m}$, às 14 horas. A mediana da ECC foi de 574,0 $(551,0 ; 602,5) \mu \mathrm{m}$ e 545,0 $(523,2 ; 569,0) \mu \mathrm{m}$, respectivamente às 6 e 14 horas $(p<0,001)$. A ECC decresceu ao longo do teste das 6 horas, sendo o decréscimo estatisticamente significativo nos momentos 30 e 60 . No teste das 14 horas a ECC manteve-se estável ao longo do teste. No entanto os valores de ECC nos momentos 30 e 60 foram semelhantes nos 2 testes (Tabela 5).

Tabela 5: Mediana (Md) e quartís (Q2) dos valores da espessura central da córnea $(\mathrm{m} \mu)$ ) em diversos momentos dos 2 testes de sobrecarga hídrica

\begin{tabular}{cccc}
\hline TSH & M 0 & M 30 & M 60 \\
\hline $\mathbf{6 h}$ & $574,0(551,0 ; 602,5) \mathbf{a}$ & $538,5(526,0 ; 571,5) \mathbf{b}$ & $550,5(532,0 ; 575,0) \mathbf{c}$ \\
$\mathbf{1 4 h}$ & $545,0(523,2 ; 569,0) \mathbf{a}$ & $542,0(528,2 ; 576,5) \mathbf{a}$ & $546,0(524,7 ; 573,5) \mathbf{a}$ \\
\hline $\boldsymbol{P}$ & $<0,001$ & $0,773^{(1)}$ & $0,050^{(1)}$ \\
\hline
\end{tabular}

Letras diferentes indicam $\mathrm{p}<0,05$ pelo teste de Wilcoxon para amostras dependentes.

$\left({ }^{1}\right)$ Comparação entre os 2 TSH pelo teste de Wilcoxon.

M O- momento zero- imediatamente antes da ingestão da água

M 30-(momento 30)- 30 minutos após a ingestão da água

M 60-(momento 60)- 60 minutos após a ingestão da água 


\section{DISCUSSÃO}

A PIO é fator de risco bem estabelecido para o desenvolvimento e para a progressão do glaucoma, e sua redução diminui o risco de conversão e progressão da doença ${ }^{5,7,12,13-15,19,22,32,62}$.

O conhecimento do verdadeiro comportamento da PIO, ao longo do tempo, é bastante limitado ${ }^{10}$ e existem controvérsias sobre qual o fator da PIO seria mais importante para a progressão glaucomatosa: PIO média? Pico de PIO? Flutuação de PIO?

Foi demonstrado que não apenas a PIO média é importante na progressão ${ }^{5,9,16}$, mas também pico e flutuação $0^{5,19-22,24,2538,57}$. Diversos estudos demonstraram que pacientes com PIO aparentemente controlada nos horários de consultório ${ }^{23,37}$, podem apresentar progressão da doença.

A melhor maneira de detectar pico e flutuação até o presente momento é a CTD, que costuma ser realizada com 7 medidas com intervalo de $3 / 3 \mathrm{~h}$, eliminando a medida das 3 horas da manhã ${ }^{51}$. Devido à dificuldade de sua realização, outros métodos mais simples, como o TSH, têm sido utilizados para avaliação de pico e flutuação da $\mathrm{PIO}^{11,40,42,44,45,60}$.

Diversos estudos mostram correlação dos valores de pico e flutuação de PIO do TSH e da minicurva ${ }^{11,41,42,50,57}$. Porém não foi encontrado na literatura estudo que avalie o melhor horário para realização do TSH. 
No presente estudo, que compara pico e flutuação de PIO em 2 TSH realizados em dois horários diferentes, nos mesmos pacientes e no mesmo dia, foi observado pico de PIO em ambos os TSH, após 30 minutos da ingestão da água. Essa observação está de acordo com o momento de elevação da PIO de outros estudos que realizaram TSH em pacientes com glaucoma em diferentes estágios e sob diferentes tratamentos ${ }^{43,61-63}$.

Não foi observada diferença entre o valor dos picos, com média de 17,34 e $16,79 \mathrm{mmHg}$, respectivamente de manhã e à tarde. Estudo que realizou 3 $\mathrm{TSH}$, com finalidade de estudar a reprodutibilidade, em 15 pacientes glaucomatosos, sem uso de medicação, também observou média de pico de PIO semelhante nos 3 horários, que foi de 25,07, 25,13, 24,93 mmHg, respectivamente, às $7,12,17$ horas $^{58}$. Em estudo mais antigo, Helal ${ }^{42}$, que também realizou TSH em horários diferentes do dia, concluiu que o pico da PIO é independente do horário da realização. No entanto, o número de pacientes de Helal ${ }^{42}$ era bastante limitado, onde 9 pacientes realizaram o TSH pela manhã e 2 pacientes (diferentes dos 9 anteriores) realizaram o TSH à tarde, o que limita extremamente as conclusões observadas ${ }^{42}$. A média dos valores de pico deste trabalho, em ambos os $\mathrm{TSH}$, foi relativamente baixa em comparação com outros estudos ${ }^{41,42,46,58,60}$, pois os pacientes estavam em uso de hipotensores oculares. Tanto os pacientes dos estudos de Medina et al que eram pacientes com glaucoma ${ }^{58}$ e Helal $^{42}$ (pacientes suspeitos de glaucoma) estavam sem uso de colírios hipotensores. 
A mediana da flutuação da PIO, observada neste trabalho em ambos os TSH, foi semelhante: $5 \mathrm{mmHg}$. O único estudo que também analisou a variação da PIO em mais de um TSH, não avaliou a flutuação da PIO da forma como foi definida neste estudo e sim o pico da PIO menos a PIO basal, a média desta variação foi de $5,54,4,40$ e $4,87 \mathrm{mmHg}$, respectivamente às $7,12,17 \mathrm{~h}$, no grupo portador de glaucoma, essa diferença não foi estatisticamente significante ${ }^{58}$. No trabalho de $\mathrm{Helal}^{42}$, que realizou um único TSH em horário diferente, sendo 9 pacientes pela manhã e 2 pacientes à tarde, a média da variação foi de 5,54 $\mathrm{mmHg}$. No presente estudo foi também esta variação da PIO (diferença entre pico menos a PIO basal), e os valores foram diferentes: $2 \mathrm{mmHg}$ e $4 \mathrm{mmHg}$, respectivamente, no TSH das 6 horas e 14 horas, porém a diferença não foi significativa. (tabela 3). A comparação com a literatura fica difícil, por diferenças na metodologia estatística, e também diferenças no tratamento.

Neste estudo, a PIO basal pela manhã $(14,31 \mathrm{mmHg})$ foi mais baixa que em outros estudos, $18 \mathrm{mmHg}^{55}, 20,67 \mathrm{mmHg}^{58}$, porém essas diferenças provavelmente ocorreram por diferenças amostrais dos estudos. No teste realizado à tarde, nos mesmos pacientes, a PIO basal foi significativamente mais baixa que a da manhã, $12,72 \mathrm{mmHg}$ e assemelha-se aos valores encontrados em outros estudos que realizaram o TSH à tarde: $13,9 \mathrm{mmHg}^{62}$, $11,1 \mathrm{mmHg}^{43}, 14,2 \mathrm{mmHg}^{64}, 12,7 \mathrm{mmHg}^{57}$. Apesar da PIO basal ter sido mais alta pela manhã ${ }^{57}$, do que à tarde, os resultados deste estudo sugerem que o valor do pico independe do valor da PIO basal. No estudo de Medina et $\mathrm{al}^{58}$, chama atenção o fato da PIO basal ser semelhante em todos os horários, tanto nos glaucomatoso, como pacientes saudáveis o que não seria esperado. 
A avaliação da ECC neste estudo mostrou valores maiores pela manhã o que poderia justificar os valores de PIO mais altos neste horário. Este achado está de acordo com Hamilton et a ${ }^{65}$ que estudaram a ECC em paciente jovens saudáveis e demonstraram que a ECC foi maior às 7 horas e diminui rapidamente ao longo de 2 horas, permanecendo estável ao longo do dia. A ECC decresceu ao longo do teste, desta forma a ECC, não poderia explicar os valores de elevação da PIO que ocorreram após 30 minutos da ingestão da água, mostrando não haver correlação entre elevação da PIO e ECC.

Ao contrário do que se poderia esperar, os picos e flutuação de PIO foram semelhantes em ambos os testes. Ao realizar o TSH em diferentes horários, observou-se que embora pela manhã a PIO apresentava valores maiores, a média dos valores dos picos de PIO foi semelhante nos dois TSH.

\section{CONCLUSÕES}

Pico e flutuação da PIO, no TSH da manhã e da tarde apresentaram valores semelhantes. Conclui-se assim que o TSH pode ser realizado em qualquer horário do dia. A elevação da pressão intra-ocular do TSH não está relacionada com alterações de ECC. 


\section{REFERÊNCIAS BIBLIOGRÁFICAS}

59. Quigley HA, Broman AT. The number of people with glaucoma worldwide in 2010 and 2020. Br J Ophthalmol. 2006;90:262-7.

60.Shields MB. Epidemiologia do glaucoma In: Shields MB. Tratado de Glaucoma. 5 ed. Rio de Janeiro: Cultura médica; 1998. p.191-2.

61. Mello PAAM, Mandia Jr C. $2^{\circ}$ Consenso Brasileiro de Glaucoma Primário de Ângulo Aberto. São Paulo: Sociedade Brasileira de Glaucoma; 2005.p.9.

62.Sommer A, Intraocular pressure and glaucoma. Am J Ophthalmol. 1989:107:186-8.

63. Bergea B, Bodin L, Svedbergh B. Impact of intraocular pressure regulation on visual fields in open angle- glaucoma. Ophthalmology. 1999;106:9971004.

64. AGIS Investigators. The Advanced Glaucoma Intervention Study (AGIS): 7. The relationship between control of intraocular pressure and visual field deterioration. Am J Ophthalmol. 2000;130:429-40.

65. Leske MC, Connell MB, Wu S, Hyman LG, Schachat AP. Risk factors for open-angle glaucoma. Arch Ophthalmol.1995;113:918-24. 
66. Sommer A, Tielsch JM, Katz J, Quigley HA, Gottsch JD, Javitt J, Singh K. Relationship between intraocular pressure and primary open angle glaucoma among white and black americans. The Baltimore Eye Survey. Arch Ophthalmol. 1991;109:1090-5.

67. Mao LK, Stewart WC, Shields B. Correlation between intraocular pressure control and progressive glaucomatous damage in primary open- angle glaucoma. Am J Ophthalmol. 1991;111:51-5.

68. Palmberg $P$. What is about pressure that really matthers in glaucoma? Ophthalmology. 2007;114:203-4.

69. Malerbi FK, Hatanaka M, Vessani RM, Susanna R Jr. Intraocular pressure variability in patients who reached target intraocular pressure. $\mathrm{Br} J$ Ophthalmol. 2005;89:540-2.

70. Heijl A, Leske MC, Bengtsson B, Hyman L, Bengtsson B, Hyman L, et al. Reduction of intraocular pressure and glaucoma progression: results from the Early Manifest Glaucoma Trial. Arch Ophthalmol. 2002; 120:1268-79.

71. European Glaucoma Prevention Study (EGPS) Group. Predictive factors for open -angle glaucoma among pacients with ocular hypertension in the European Glaucoma Prevention Study. Ophthalmology. 2007;114:3-9. 
72. Leske MC, Heijl A, Hyman L, Bengtsson B, Dong L, Yang Z. Predictors of long-term progression in the early manifest glaucoma trial. Ophthalmology $2007 ; 114: 1965-72$.

73. Collaborative Normal-tension glaucoma study group. The effectiveness of intraocular pressure reduction in the treatment of normal-tension glaucoma. Am J Ophthlmol. 1998;126:498-505.

74. O'Brien C, Schartz B, Takamoto T, Wu DC. Intraocular pressure and the rate of visual field loss in chronic open-angle glaucoma. Am J Ophthlamol. 1991;111:491-500.

75. Gordon MO, Beiser JA, Brandt JD, Heuer DK, Higginbotham EJ, Johnson CA, et al. The ocular hypertension treatment study. Baseline factors that predict the onset of primary open-angle glaucoma. Arch Ophthalmol. 2002;120:714-20.

76. Jonas JB, Budde W, Stroux A, Oberacher-Velten IM, Junemann A. Single intraocular pressure measurement and diurnal intraocular pressure profiles. Am J Ophthalmol. 2005;139:1136-7.

77. Medeiros FA, Weinreb RN, Zangwill LM, Alencar LM, Sample PA, Vasile C, et al. Long-term intraocular pressure fluctuations and risk of conversion from ocular hypertension to glaucoma. Ophthalmology. 2008;115:934-40. 
78. Asrani S, Zeimer R, Wilensky J, Gieser D, Vitale S, Lienmuth K. Large diurnal fluctuations in intraocular pressure are an independent risk factor in patients with glaucoma. J Glaucoma. 2000;9:134-42.

79. Caprioli J, Coleman AL. Intraocular pressure fluctuation. Ophthalmology. 2008;115:1123-9.

80. Nouri- Mahdavi K, Hoffman BA, Coleman AL, Liu G, Li G, Gaasterland D, Caprioli J. Peditctive Factors for glaucomatous visual field progression in the Advanced Glaucoma Intervention Study. Ophthalmology. 2004; 111: 1627-35.

81. Sacca SC, Rolando M, Marletta A, Macrí A, Cerqueti P, Ciurlo G. Fluctuations of intraocular pressure during the day in open-angle glaucoma, normal tension glaucoma and normal subjects. Ophthalmologica. $1998 ; 212: 115-9$

82. Bengtsson B, Leske MC, Hyman L, Heijil A. Fluctuation of intraocular pressure and glaucoma progression in the early manifest glaucoma trial. 2007. Ophthalmology. 2007:144:205-9.

83. Singh K, Shrivastava A. Intraocular pressure fluctuations: how much do they matter? Curr Opin Ophthalmol. 2009;20:84-7.

84.Susanna R Jr, Weinreb RN. Glaucoma: perguntas e respostas. Rio de Janeiro: Cultura Médica;2005. 
85. Rota-Bartelink AM, Pitt A, Story I. Influence of diurnal variation on the intraocular pressure measurement of treated primary open-angle glaucoma during office hours. J Glaucoma. 1996;5:410-15.

86. Rodrigues LD, Silva, M R B M, Schellini SA, Jorge, EN. Picos de pressão intra-ocular: comparação entre curva tensional diária, minicurva e medida da pressão intra-ocular às 6 horas. Arq Bras Oftalmol. 2004; 67:127-31.

87. Liu JHH, Sit AJ, Weinre RN. Variation of 24-hour intraocular pressure in healthy individuals. Ophthalmology. 2005;112:1670-5.

88. Mosaed S, Liu JHK, Weinreb RN. Correlation between office and peak nocturnal intraocular pressure in healthy subjects and glaucoma pacients. Am J Ophthlmol. 2005;139:320-4.

89. Dinn RB, Zimmerman B, Shuba LM, Doan AP, Maley MK, Greenlee EC, et al. Concordance of diurnal intraocular pressure between fellow eyes in primary open-angle glaucoma. Ophthalmology. 2007; 114:915-20.

90.Zeimer RC, Wilensky J T, Gieser DK. Presence and rapid decline of early morning intraocular pressure peaks in glaucoma pacients. Ophthalmology 1990;97:547-50.

91. Waisberg Y. Medida das seis horas da manhã, da curva diária de pressão intra-ocular: quando ela é necessária? Rev Bras Oftalmol. 1982;41:34-50. 
92. David R, Zangwill L, Briscoe D, Dagan M, Yagev R, Yassur Y. Diurnal intraocular pressure variations: an analysis of 690 diurnal curves. $\mathrm{Br} \mathrm{J}$ Ophthalmol. 1992;76:280-3.

93. De Moraes CG, Prata TS, Tello C, Ritch R. Glaucoma with early visual field loss affecting both hemifields and risk of disease progression. Arch Ophthalmol. 2009;127:1129-34.

94. Fogagnolo P, Rossetti L, Mazzolani F, Orzalesi N. Circadian variations in central corneal thickness and intraocular pressure in patients with glaucoma. $\mathrm{Br} \mathrm{J}$ of Ophthalmol. 2006;90:24-8.

95. Barkana Y, Anis S, Liebmann J, Tello C, Ritch R. Clinical Utility of intraocular pressure monitoring outside of normal office hours in patient with glaucoma. Arch Opthalmol. 2006;124:793- 7.

96. Konstas AGP, Topozis F, Leliopoulou O, Pappas T, Georgiadis N, Jerkins $\mathrm{JN}$, et al. 24-hour intraocular pressure control with maximum medical therapy compared with surgery in patients with advanced open-angle glaucoma. Ophthalmology. 2006;113:761-5.

97. Hughes E, Spry P, Diamond J. 24-Hour monitoring of intraocular pressure in glaucoma management: a retrospective review. J Glaucoma. 2003; 12: 232-6. 
98. Kumar RS, Guzman HP, MS Sung, Ong PY, Goldberg I. Does peak intraocular pressure by water drinking test reflect peak circadian levels? A pilot study. Clin Exp Ophthalmol. 2008:36:312-5.

99. Medeiros FA, Leite CA, Susanna Jr R. Correlação entre os picos pressóricos da curva tensional diária e o teste de sobrecarga hídrica. Rev Bras Oftalmol. 2001;60:418-23.

100. Helal Jr J. Contribuição ao estudo da pressão intra-ocular: picos de pressão intra-ocular na curva diária de pressão e na prova de sobrecarga hídrica. Rev Bras Oftalmol. 1988;47:7-12.

101. Danesh- Meyer HV, Papchenko FT, Tan Y, Gamble GD. Medically controlled glaucoma patients show greater increase in intraocular pressure than surgically controlled patients with the water drinking test. Ophthalmology. 2008;9:1566-70.

102. Danesh- Meyer HV. The water-drinking test the elegance of simplicity. Clin Exp Ophthalmol. 2008;36:301-3.

103. Susanna R Jr, Vessani R M, L. O teste de sobrecarga hídrica no manuseio do glaucoma. Rev Bras Oftalmol. 2007;66:142-5.

104.

Carvalho CA, Helal Jr J. Valor da prova de sobrecarga hídrica no seguimento de pacientes com glaucoma crônico simples em tratamento. Rev Bras Oftalmol. 1988;47:13-6. 
105. Fan CJ, McGhee CNJ. Publication and citation in Ophthlalmology: glaucoma and the water-provocation test- wring out the old and ring in the new? Clin Exp Ophthalmol. 2008;36:304-5.

106. Roetth A Jr. Effect of changes in osmotic pressure of blood on aquous humor dynamics A.M. A. Arch Ophthalmol.1954;52:571-82.

107. Moraes VD, Reis ASC, Cavalcante AFS, Sano ME, Susanna Jr R. Choroidal expansion during the water drinking test. Graefes Arch Clin Exp Ophthalmol. 2009;247:385-9

108. Frota ACA, Meirelles SHS, Ávila E, Yamane Y. Correlação entre picos pressóricos de teste de sobrecarga hídrica e curva ambulatorial de presssão intra-ocular em glaucoma primário de ângulo aberto suspeito. Rev Bras Oftalmol. 2005; 64:383-7.

109. Meirelles SHS, Yamane R, Alvares RM, Botelho PB, Morais FB, Moreira $\mathrm{PB}$, et al. Estudo comparativo entre a curva tensional diária de pressão intra-ocular e a associação da curva ambulatorial com o teste de sobrecarga hídrica no glaucoma primário de ângulo aberto, glaucoma de pressão normal e olhos normais. Arq Bras Oftalmol. 2007;70:471-9.

110. Leydhecker W. Evaluation of the water-drinking test. $\mathrm{Br} \mathrm{J}$ Ophthalmol. 1954;38: 290-4.

111. Winder AF, Siddiqui AA, Donovan HC. Ocular hypertension and systemic responses to the water-drinking test. $\mathrm{Br} \mathrm{J}$ Ophthalmol. 1987; 62:414-9. 
112. Roth JA. Inadequate diagnostic value of the water-drinking test. $\mathrm{Br} \mathrm{J}$ Ophthalmol. 1974;58:55-61.

113. Susanna R Jr, Medeiros FA, Vessani RM, Giampani J Jr, Borges AS, Jordão ML. Intraocular pressure fluctuations in response to the waterdrinking provocative test in patients using latanoprost versus usoprostone. $\mathrm{J}$ Ocul Pharmacol Ther. 2004;20:401-10.

114. Meirelles SHS, Mendes KRR, Álvares RM, Villela ACM, Lucena DAP, Yamane R. Influência do peso corporal e do índice de massa corporal no teste de sobrecarga hídrica. Rev Bras Oftalmol. 2008; 67:132-7.

115. Susanna R Jr, Vessani RM, Sakata L, Zacarias LC, Hatanaka M. The relation between intraocular pressure peak in the water drinking test and visual field progression in glaucoma. Br J Ophthalmol. 2005;89:1298-301.

116. Medina FMC, Rodrigues FKP, Filho PTPP, Matsuo T, Vascocellos JPC, Costa VP. Reproducibility of water drinking test performed at different times of the day. Arq Bras Oftalmol. 2009;72: 283-90.

117. Lichter PR, Musch DC, Gillespie BW, Guire KE, Janz NK, Wren PA, et al. Interin clinical outcomes in the collaborative initial glaucoma treatment Study Comparing initial treatment randomized to medications or Surgery. Ophthalmology. 2001;108:1943-53. 
118. Susanna R Jr, Medeiros FA, Vessani RM, Giampani J Jr, Borges AS, Jordão ML. Intraocular pressure fluctuations in response to the waterdrinking provocative test in patients using latanoprost versus usoprostone. $\mathrm{J}$ Ocul Pharmacol Ther. 2004;20:401-10.

119. Roth JA. Inadequate diagnostic value of the water-drinking test. $\mathrm{Br} \mathrm{J}$ Ophthalmol. 1974;58:55-61.

120. Susanna R Jr, Hatanaka M, Vessani R M, Pinheiro A, Morita C. Correlation of asymetric glaucomatous visual field and water-drinking test response. Invest Ophthalmol Vis Sci. 2006;47:641-4.

121.

Drance SM. Studies with applanation water tests. Arch Ophthalmol.1963; 69:39- 43.

122. Guedes RAP, Guedes VMP, Chaoubah A. Application du test de surcharge hydrique après sclérectomie profunde non perfurante. $\mathrm{J} F r$ Ophthalmol. 2005; 28:1076-80.

123. Hamilton KE, Optom B, Pye DC, Optom M, Aggarwalla S, Evian S, et al. Diurnal variation of central corneal thickness and Goldman applanation tonometry estimates of intraocular pressure. J Glaucoma. 2007;16:29-35. 
Anexos 
Anexo 1 - Comitê de Ética em Pesquisa.

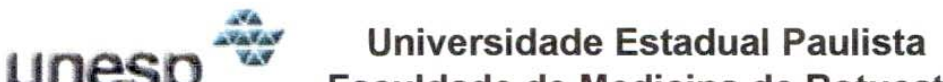 Faculdade de Medicina de Botucatu}

Distrito Rubião Junior, $s / n^{\circ}$ - Botucatu - S.P.

CEP: 18.618-970

Fone/Fax: (0xx14) 3811-6143

e-mail secretaria: capellup@fmb.unesp.br

Registrado no Ministério da Saúde em 30 de abril de 1997

Botucatu, 02 de abril de 2.007

OF. 70/2007-CEP

Ilustríssima Senhora

Prof ${ }^{u}$. Dr ${ }^{a}$. Maria Rosa Bet Moraes Silva

Departamento de Oftalmo e Otorrino da

Faculdade de Medicina de Botucatu

Prezado Dr ${ }^{a}$ Maria Rosa,

De ordem da Senhora Coordenadora deste CEP, informo que o Projeto de Pesquisa "Correlação da pressão intra-ocular de 24 horas e do teste de sobrecarga híbrica em pacientes portadores de glaucoma e hipertensão intra-ocular", a ser conduzido por Mariluci Tosi, orientada por Vossa Senhoria, recebeu do relator parecer favorável, aprovado em reunião de 02 de abril de 2.007.

Situação do Projeto: APROVADO.

OBS: Ao final da execução deste projeto, deverá ser apresentado ao CEP "Relatório Final de Atividades".

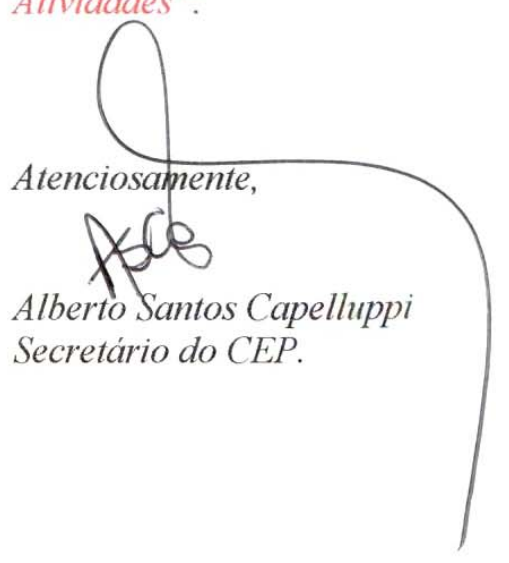




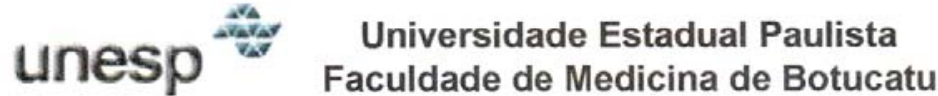

Distrito Rubião Junior, $s / n^{\circ}$ - Botucatu - S.P.

CEP: $18.618-970$

Fone/Fax: $(0 \times x 14) 3811-6143$

e-mail secretaria: capellup@fmb.unesp.br

Ilustríssima Senhora

Profa. Dra. Maria Rosa Bet Moraes Silva

Departamento de Oftalmologia e Otorrinolaringologia

Faculdade de Medicina de Botucatu

Prezada Profa. Maria Rosa,

De ordem da Senhora Coordenadora deste CEP informo que em 23/1 1/2007 foi autorizada a inclusão de "mais um teste de sobrecarga hidrica (TSH) em horário diferente" ao Projeto de Pesquisa "Correlaçāo da pressão intra-ocular de 24 horas e do teste de sobrecarga híbrica em pacientes portadores de glaucoma e hipertensāo intra-ocular", que está sendo conduzido por Mariluci Tosi, orientada por Vossa Senhoria, aprovado por este CEP 02 de abril de 2.007.

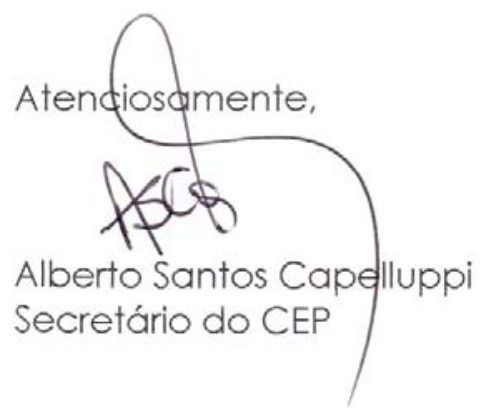

